\title{
Cardiomyocyte BRAF and type 1 RAF inhibitors promote cardiomyocyte and cardiac hypertrophy in mice in vivo
}

Article

Published Version

Creative Commons: Attribution 4.0 (CC-BY)

Open Access

Clerk, A. ORCID: https://orcid.org/0000-0002-5658-0708, Meijles, D. N., Hardyman, M. A., Fuller, S. J., Chothani, S. P., Cull, J. J., Cooper, S. T. E., Alharbi, H. O., Vanezis, K., Felkin, L. E., Markou, T., Leonard, S. J., Shaw, S. W., Rackham, O. J. L., Cook, S. A., Glennon, P. E., Sheppard, M. N., Sembrat, J. C., Rojas, M., McTiernan, C. F., Barton, P. J. and Sugden, P. H. (2022) Cardiomyocyte BRAF and type 1 RAF inhibitors promote cardiomyocyte and cardiac hypertrophy in mice in vivo. Biochemical Journal, 479 (3). pp. 401-424. ISSN 02646021 doi: https://doi.org/10.1042/BCJ20210615 Available at https://centaur.reading.ac.uk/99825/

It is advisable to refer to the publisher's version if you intend to cite from the work. See Guidance on citing.

To link to this article DOI: http://dx.doi.org/10.1042/BCJ20210615

Publisher: Portland Press Limited

All outputs in CentAUR are protected by Intellectual Property Rights law, including copyright law. Copyright and IPR is retained by the creators or other copyright holders. Terms and conditions for use of this material are defined in 
the End User Agreement.

www.reading.ac.uk/centaur

\section{CentAUR}

Central Archive at the University of Reading

Reading's research outputs online 


\title{
Cardiomyocyte BRAF and type 1 RAF inhibitors promote cardiomyocyte and cardiac hypertrophy in mice in vivo
}

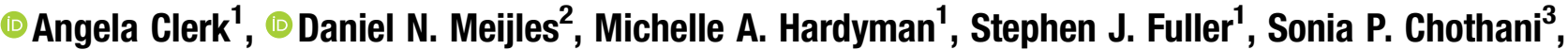 \\ Joshua J. Cull ${ }^{1}$, Susanna T.E. Cooper ${ }^{2}$, Hajed 0. Alharbi ${ }^{1}$, Konstantinos Vanezis ${ }^{4,6, \star}$, Leanne E. Felkin ${ }^{4,5}$, \\ Thomais Markou', Samuel J. Leonard' ${ }^{1}$, Spencer W. Shaw ${ }^{1}$, Owen J.L. Rackham ${ }^{3}$, Stuart A. Cook ${ }^{3,6,7}$, \\ Peter E. Glennon ${ }^{8}$, Mary N. Sheppard ${ }^{9}$, John C. Sembrat ${ }^{10}$, Mauricio Rojas ${ }^{10}$, Charles F. McTiernan ${ }^{11}$, \\ Paul J. Barton ${ }^{4,5}$ and Peter H. Sugden ${ }^{1}$
}

\begin{abstract}
${ }^{1}$ School of Biological Sciences, University of Reading, Reading, U.K.; ${ }^{2}$ Molecular and Clinical Sciences Institute, St. George's University of London, London, U.K.; ${ }^{3}$ Program in Cardiovascular and Metabolic Disorders, Duke-National University of Singapore Medical School, Singapore City, Singapore; ${ }^{4}$ National Heart and Lung Institute, Imperial College London, London, U.K.; ${ }^{5}$ Cardiovascular Research Centre, Royal Brompton and Harefield Hospitals, London, U.K.; ${ }^{6}$ MRC London Institute of Medical Sciences, Imperial College London, London, U.K.; ${ }^{7}$ National Heart Centre Singapore, Singapore City, Singapore; ${ }^{8}$ University Hospitals Coventry and Warwickshire, University Hospital Cardiology Department, Clifford Bridge Road, Coventry, U.K.; ${ }^{9}$ CRY Cardiovascular Pathology Department, St. George's Healthcare NHS Trust, London, U.K.; ${ }^{10}$ Division of Pulmonary, Allergy and Critical Care Medicine, and Dorothy P \& Richard P Simmons Center for Interstitial Lung Disease, Department of Medicine, University of Pittsburgh, Pittsburgh, U.S.A; ${ }^{11}$ Heart, Lung, Blood Vascular Medicine Institute, University of Pittsburgh, Pittsburgh, U.S.A
\end{abstract}

Correspondence: Angela Clerk (a.clerk@reading.ac.uk)

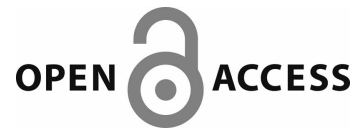

${ }^{*}$ Current address MiNA

Therapeutics Ltd, London, U.K.

Received: 11 August 2021 Revised: 12 January 2022 Accepted: 27 January 2022

Accepted Manuscript online: 27 January 2022

Version of Record published: 11 February 2022
The extracellular signal-regulated kinase 1/2 (ERK1/2) cascade promotes cardiomyocyte hypertrophy and is cardioprotective, with the three RAF kinases forming a node for signal integration. Our aims were to determine if BRAF is relevant for human heart failure, whether BRAF promotes cardiomyocyte hypertrophy, and if Type 1 RAF inhibitors developed for cancer (that paradoxically activate ERK1/2 at low concentrations: the 'RAF paradox') may have the same effect. BRAF was up-regulated in heart samples from patients with heart failure compared with normal controls. We assessed the effects of activated BRAF in the heart using mice with tamoxifen-activated Cre for cardiomyocytespecific knock-in of the activating V600E mutation into the endogenous gene. We used echocardiography to measure cardiac dimensions/function. Cardiomyocyte BRAF ${ }^{V 600 E}$ induced cardiac hypertrophy within $10 \mathrm{~d}$, resulting in increased ejection fraction and fractional shortening over 6 weeks. This was associated with increased cardiomyocyte size without significant fibrosis, consistent with compensated hypertrophy. The experimental Type 1 RAF inhibitor, SB590885, and/or encorafenib (a RAF inhibitor used clinically) increased ERK1/2 phosphorylation in cardiomyocytes, and promoted hypertrophy, consistent with a 'RAF paradox' effect. Both promoted cardiac hypertrophy in mouse hearts in vivo, with increased cardiomyocyte size and no overt fibrosis. In conclusion, BRAF potentially plays an important role in human failing hearts, activation of BRAF is sufficient to induce hypertrophy, and Type 1 RAF inhibitors promote hypertrophy via the 'RAF paradox'. Cardiac hypertrophy resulting from these interventions was not associated with pathological features, suggesting that Type 1 RAF inhibitors may be useful to boost cardiomyocyte function.

\section{Introduction}

Mammalian cardiomyocytes (the contractile cells of the heart) withdraw from the cell cycle in the perinatal period and become terminally differentiated [1]. However, the adult heart is subjected to pathophysiological stresses that increase workload and require greater cardiac output. To 
accommodate this, cardiomyocytes undergo hypertrophic growth (increasing in size in the absence of proliferation), increasing and adapting the myofibrillar apparatus, leading to cardiac hypertrophy. In some instances, this is a benign adaptation to, for example, pregnancy or endurance exercise [2]. Such 'physiological' hypertrophy is generally reversible once the stress has ceased, and is not associated with fibrosis. In chronic conditions (e.g. hypertension), or with acute damage to the heart (e.g. a result of myocardial infarction), the heart initially adapts to maintain cardiac output with cardiomyocyte hypertrophy, but this adaptive hypertrophy is not sustained. Contractile function becomes compromised and heart failure develops, with increased cardiomyocyte cell death [3], loss of capillaries [4], inflammation and fibrosis [5].

The extracellular signal-regulated kinase 1/2 (ERK1/2) cascade is the prototypic mitogen-activated protein kinase (MAPK) pathway, first identified in the context of its role in promoting cell division in proliferating cells. However, this pathway also promotes cardiomyocyte hypertrophy and is cardioprotective [6-8]. ERK1/2 operate in a cascade in which RAF kinases (ARAF, BRAF, RAF1) phosphorylate and activate MAPK kinases $1 / 2$ (MKK1/2), and these phosphorylate/activate ERK1/2 [9]. RAF kinases receive inputs from different stimuli and are regulated at multiple levels [9]. Activation requires interaction with activated Ras and, in proliferating cells, this promotes formation of active RAF homo- or heterodimers. RAF kinases are subject to both activating and inhibitory phosphorylations. In particular, RAF1 requires phosphorylation of Ser338/Tyr341 for activity (all phosphorylation sites refer to the human protein; equivalent residues are present in the mouse and rat proteins). BRAF has high basal activity because it possesses acidic residues in this domain, but is phosphorylated on Ser445 [equivalent to RAF1(Ser338)].

The regulation of RAF kinases in terminally differentiated cardiomyocytes is less well understood. RAF1 and ARAF are activated in cardiomyocytes by growth factors and hypertrophic agonists such as endothelin-1 [10]. As in proliferating cells, RAF1 activation requires activation of the small G protein, Ras, and this leads to phosphorylation of RAF1(Ser338) [11,12]. Cardiomyocyte-specific expression of dominant-negative RAF1 in mice increases cardiomyocyte apoptosis, with cardiomyopathy developing in response to pressure-overload $[13,14]$, consistent with a cardioprotective role of RAF1. BRAF is expressed in cardiomyocytes [15], but there is little information on its role in the heart.

Activating mutations in the ERK1/2 cascade can cause cancer. With oncogenic mutations in BRAF being associated with $\sim 30 \%$ of all cancers [16], there has been great emphasis on developing BRAF inhibitors, some of which (e.g. dabrafenib, encorafenib) are in clinical use $[17,18]$. These target the highly oncogenic BRAF ${ }^{\mathrm{V} 600 \mathrm{E} / \mathrm{K}}$ mutated enzyme, but also inhibit wild-type enzymes (e.g. dabrafenib has $\mathrm{IC}_{50}$ values of 5.2 and $6.3 \mathrm{nM}$ for BRAF and RAF1, respectively [19]). This first generation of drugs are Type 1 or 1.5 inhibitors that interact with the ATP binding site, binding to and locking the enzyme in an active conformation. These drugs revealed a 'RAF paradox', in which, rather than inhibiting the pathway, they activate ERK1/2 at low concentrations [20]. This is potentially because inhibitor binding to one partner in a RAF dimer locks the other in an active conformation and (because the inhibitor is at a non-saturating concentration) this protomer activates MKK1/2 in the presence of activated Ras. Overall, the BRAF inhibitors used for cancer could affect wild-type RAF signalling in noncancerous cells, potentially in unpredictable ways. Indeed, our recent study showed that dabrafenib (a Type 1.5 inhibitor [19]) has an anti-fibrotic effect in hypertension-induced cardiac hypertrophy in mice [21].

Here, we show that BRAF expression is up-regulated in human heart failure. Activation of cardiomyocyte BRAF was sufficient to promote hypertrophy in mice, whilst the Type 1 RAF inhibitor, SB590885, and encorafenib (in clinical use) activated ERK1/2 signalling in cardiomyocytes causing hypertrophy in cultured cells. Both drugs induced cardiac hypertrophy in mouse hearts in vivo. Interestingly, cardiac hypertrophy induced by activation of cardiomyocyte BRAF or these RAF inhibitors was not associated with any overt fibrosis or cell infiltration.

\section{Materials and methods Human heart samples}

Failing human tissue sample collections were obtained from patients who consented to a protocol reviewed and approved by the University of Pittsburgh Institutional Review Board. Patient information is provided in Supplementary Table S1. Transmural tissue at the level of the anterior papillary muscle was collected at the time of cardiac transplantation from the left ventricle of end-stage heart failure patients. Samples were collected in the operating room and transported in ice-cold St. Thomas' cardioplegia solution, flash frozen within $20 \mathrm{~min}$ of excision, and stored at $-80^{\circ} \mathrm{C}$ prior to utilisation. Non-failing heart samples were collected under 
University of Pittsburgh CORID \#451 (Committee for Oversight of Research and Clinical Training Involving Decedents) and with consent being obtained by the local Organ Procurement Organisation (OPO), CORE (Center for Organ Recovery and Education). Control left ventricular tissues were collected from hearts that were rejected for transplant for varying reasons. Tissues were collected and stored in a similar manner as the failing hearts with between $20-45$ min of time elapsing between cross-clamp and freezing of the tissue.

\section{Bioinformatics analysis for transcript expression in dilated cardiomyopathy}

mRNA expression of BRAF (ENSG00000157764), ARAF (NSG00000078061) and RAF1 (ENSG00000132155) in control and diseased human hearts was determined using a previously published RNASeq dataset derived from left ventricular samples of 97 patients with end-stage dilated cardiomyopathy taken at the time of transplantation or left ventricular assist device implantation, together with 108 non-diseased controls, as described in Heinig et al. [22]. Differential expression analysis was carried out using DESeq2 (V1.18.1, Wald test) [23] between dilated cardiomyopathy patient samples and control samples.

\section{Ethics statement for animal experiments}

Animals were housed at the BioResource Unit at University of Reading (U.K. registered with a Home Office certificate of designation). All procedures were performed in accordance with U.K. regulations and the European Parliament Directive 2010/63/EU for animal experiments. Work was undertaken in accordance with local institutional animal care committee procedures (University of Reading) and the U.K. Animals (Scientific Procedures) Act 1986 (Procedure Project Licences 70/8248, 70/8249, and P8BAB0744).

\section{Animal housing, husbandry and welfare}

Adult mice and rats were allowed to acclimatise for at least $7 \mathrm{~d}$ prior to experiments. Neonatal rats were culled by cervical dislocation for which additional approval and licences are not required according to U.K. regulations. Mice were housed in Tecniplast IVC cages (total area $512 \mathrm{~cm}^{2}$; maximum five mice per cage). Adult male rats were housed in open top NKP cages (total area $1632 \mathrm{~cm}^{2}$; maximum five rats per cage). Cages were supplied with aspen sawdust bedding, sizzle nesting, cardboard tunnels and housing. Additional enrichment included chew sticks and millet to encourage foraging behaviour. Animals were provided with water and food (SDS Rm3 pelleted food for mice; SDS RM3 expanded pelleted food for rats) ad libitum, with a 12:12 light/ dark cycle and room temperature of $21^{\circ} \mathrm{C}$. Breeding was conducted with mice between 6 weeks and 8 months with a maximum of six litters per female. All animals were checked at least once a day by a trained, competent person and licence holders informed of any welfare issues, with consultation with a Named Veterinary Surgeon when necessary. Mice undergoing procedures were monitored using a score sheet and routinely culled if they reached a predefined endpoint agreed with the Named Veterinary Surgeon. Weights were taken before, during and at the end of the procedures. Mouse weights from the start and end of procedures are provided in Supplementary Table S2.

Allocation of mice to specific groups was on a random basis with randomisation performed independently of the individual leading the experiment. Mice were excluded after randomisation only if there was a health problem unrelated to the experiment, in which case they were culled. One mouse (Cre ${ }^{\mathrm{MCM} /-}$, vehicle treated) was culled because of wounds inflicted as a result of aggressive behaviour. Individuals conducting the in vivo studies were not blinded to experimental conditions for welfare monitoring purposes. Data and sample analysis (e.g. echocardiography, histology) was performed by individuals who were blinded to intervention.

\section{In vivo mouse studies for cardiomyocyte-specific activation of BRAF}

Genetically modified mice were from Jackson Laboratories, imported into the U.K. and transported to University of Reading for breeding in-house. We used mice with a floxed cassette for Cre-induced knock-in of

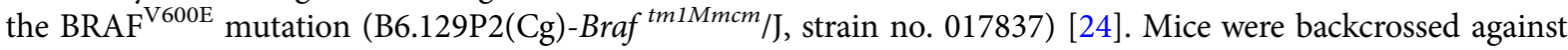
the $\mathrm{C} 57 \mathrm{Bl} / 6 \mathrm{~J}$ background at University of Reading for at least 4 generations prior to experimentation. Mice were bred with Myh6-MERCreMER mice expressing tamoxifen-inducible Cre recombinase under control of the mouse Myh6 promoter [Tg(Myh6-cre)1Jmk/J, strain no. 009074] [25]. Breeding protocols were used to produce $\mathrm{BRAF}^{\mathrm{V} 600 \mathrm{E} / \mathrm{WT}} / \mathrm{Cre}^{\mathrm{MCM} /-}$ mice, heterozygous for the $\mathrm{BRAF}^{\mathrm{V} 600 \mathrm{E}}$ allele and hemizygous for Cre. $\mathrm{Cre}^{\mathrm{MCM} /-}$ mice, hemizygous for Cre alone, were derived from crosses between the $\mathrm{Tg}(\mathrm{Myh} 6-\mathrm{cre}) 1 \mathrm{Jmk} / \mathrm{J}$ and B6.129P2(Cg)-Braf ${ }^{t m I M m c m} / \mathrm{J}$, and treated with/without tamoxifen to control for tamoxifen-induced Cre activation. 
DNA for genotyping was extracted from ear clips using Purelink genomic DNA (gDNA) mini-kits (Invitrogen) according to the manufacturer's instructions. Briefly, tissue was digested in genomic digestion buffer containing proteinase $\mathrm{K}$ (overnight, $55^{\circ} \mathrm{C}$ ). Following centrifugation $\left(12000 \times \mathrm{g}, 3 \mathrm{~min}, 18^{\circ} \mathrm{C}\right.$ ), supernatants were incubated with RNAse A ( 2 min) before addition of genomic lysis binding buffer mixed with an equal volume of ethanol. gDNA was purified using Purelink spin columns and PCR amplified with specific primers (Supplementary Table S3) using GoTaq Hot Start Polymerase (Promega). PCR conditions were $95^{\circ} \mathrm{C}$ for $3 \mathrm{~min}$, followed by up to 33 cycles of $95^{\circ} \mathrm{C}$ denaturation for $30 \mathrm{~s}, 30 \mathrm{~s}$ annealing, elongation at $72^{\circ} \mathrm{C}$ for $30 \mathrm{~s}$, followed by a $7 \mathrm{~min} 72^{\circ} \mathrm{C}$ final extension. PCR products were separated on $2 \%(\mathrm{w} / \mathrm{v})$ agarose gels $(25 \mathrm{~min}$, $80 \mathrm{~V})$ and visualised under UV light.

Male mice (8-9 weeks) were treated with a single dose of tamoxifen $(40 \mathrm{mg} / \mathrm{kg}$ i.p.; Sigma-Aldrich) or vehicle. Tamoxifen was dissolved in $0.25 \mathrm{ml}$ ethanol and then mixed with $4.75 \mathrm{ml}$ corn oil. For confirmation of recombination in the heart, RNA was extracted from heart powders and cDNA prepared as described below. cDNA $(4 \mu \mathrm{l})$ was subjected to PCR analysis using GoTaq Hot Start Polymerase with specific primers and conditions (Supplementary Table S3). PCR conditions were $95^{\circ} \mathrm{C}$ for $5 \mathrm{~min}$, followed by 40 cycles of $95^{\circ} \mathrm{C}$ denaturation for $40 \mathrm{~s}, 40 \mathrm{~s}$ annealing, elongation at $72^{\circ} \mathrm{C}$ for $60 \mathrm{~s}$, followed by a $7 \mathrm{~min} 72^{\circ} \mathrm{C}$ final extension. $50 \%$ of the resulting product was digested with XbaI (New England Biolabs) $\left(3 \mathrm{~h}, 37^{\circ} \mathrm{C}\right)$. Digested and undigested products were separated by electrophoresis on a $2 \%(\mathrm{w} / \mathrm{v})$ agarose gel $(85 \mathrm{~V}, 45 \mathrm{~min})$. BRAF ${ }^{\mathrm{V} 600 \mathrm{E}}$ knock-in introduces a novel Xbal site in the PCR product in the cDNA producing additional products [24].

\section{In vivo mouse studies for effects of SB590885 and encorafenib}

Male wild-type C57Bl/6J mice (7-8 weeks of age) were from Charles River (U.K.). Drug delivery used Alzet osmotic pumps (models 1007D or 1004; supplied by Charles River), filled according to the manufacturer's instructions in a laminar flow hood using sterile technique. Mice were treated with vehicle only [DMSO/PEG mix: 50\% (v/v) DMSO, 20\% (v/v) polyethylene glycol 400, 5\% (v/v) propylene glycol, $0.5 \%(\mathrm{v} / \mathrm{v})$ Tween 80 made up to $100 \%$ with $\mathrm{H}_{2} \mathrm{O}$ ], or $0.5 \mathrm{mg} / \mathrm{kg} / \mathrm{d}$ SB590885 (Selleck Chemicals) or $3 \mathrm{mg} / \mathrm{kg} / \mathrm{d}$ encorafenib (MedChemExpress) dissolved in DMSO/PEG mix. In some studies, mice also received a minipump containing angiotensin II (AngII) for delivery at $0.8 \mathrm{mg} / \mathrm{kg} / \mathrm{d}$ or acidified PBS vehicle. Minipumps were incubated overnight in sterile $\mathrm{PBS}\left(37^{\circ} \mathrm{C}\right)$ prior to implantation. Implantation was performed under continuous inhalation anaesthesia using isoflurane (induction at 5\%, maintenance at 2-2.5\%) mixed with $21 / \mathrm{min}_{2}$. A $1 \mathrm{~cm}$ incision was made in the mid-scapular region and mice were given $0.05 \mathrm{mg} / \mathrm{kg}$ (s.c.) buprenorphine (Ceva Animal Health Ltd.) to repress post-surgical discomfort. Minipumps were implanted portal first in a pocket created in the left flank region of the mouse. Wound closure used a simple interrupted suture with polypropylene 4-0 thread (Prolene, Ethicon). Mice were allowed to recover singly and returned to their home cage once fully recovered.

\section{Cardiac ultrasound}

Echocardiography was performed on anaesthetised mice using a Vevo 2100 imaging system equipped with a MS400 18-38 MHz transducer (Visualsonics). Mice were anaesthetised in an induction chamber with isoflurane (5\% flow rate) with $1 \mathrm{l} / \mathrm{min} \mathrm{O}_{2}$ then transferred to the heated Vevo Imaging Station. Anaesthesia was maintained with $1.5 \%$ isoflurane delivered via a nose cone. Baseline scans were taken prior to experimentation $(-7$ to -3 days). Further scans were taken at intervals following tamoxifen treatment or minipump implantation. Imaging was completed within $20 \mathrm{~min}$. Mice were recovered singly and transferred to the home cage once fully recovered. Left ventricular cardiac dimensions were assessed from short axis M-mode images with the axis placed at the mid-level of the left ventricle at the level of the papillary muscles. Data analysis was performed using VevoLAB software (Visualsonics) and data were gathered from two M-mode scans at each time point, taking mean values across four cardiac cycles. Cardiac function and global longitudinal strain were measured from B-mode long axis images using VevoStrain software for speckle tracking. Mice were culled by $\mathrm{CO}_{2}$ inhalation followed by cervical dislocation. Hearts were excised quickly, washed in PBS, dried and snap-frozen in liquid $\mathrm{N}_{2}$ or fixed for histology.

\section{Histology and assessment of myocyte size and fibrosis}

Histological staining and analysis were performed as previously described [26], assessing general morphology by haematoxylin and eosin (H\&E) and fibrosis by Masson's trichrome and picrosirius red (PSR). 


\section{Adult rat heart perfusions}

Adult male (300-350 g) Sprague-Dawley rats were anaesthetised with a lethal intraperitoneal dose of pentobarbital sodium $(60 \mathrm{mg} / \mathrm{kg})$. After complete anaesthesia was induced, heparin $(1000 \mathrm{U} / \mathrm{kg})$ was administered intravenously. The chest cavity was opened and the heart and lungs were removed into modified ice-cold KHBBS $\left(25 \mathrm{mM} \mathrm{NaHCO}_{3}, 119 \mathrm{mM} \mathrm{NaCl}, 35 \mathrm{mM} \mathrm{KCl}, 2.5 \mathrm{mM} \mathrm{CaCl}_{2}, 1.2 \mathrm{mM} \mathrm{MgSO}_{4}, 1.2 \mathrm{mM} \mathrm{KH}_{2} \mathrm{PO}_{4}\right.$ equilibrated with $\left.95 \% \mathrm{O}_{2} / 5 \% \mathrm{CO}_{2}\right)$ whilst the heart was still beating. Hearts were perfused retrogradely $\left(37^{\circ} \mathrm{C}\right.$ at $70 \mathrm{~mm}$ $\mathrm{Hg}$ ) as described previously [27] with a 15 min equilibration period. SB590885 was added at the end of the equilibration period and all perfusions continued for a further $15 \mathrm{~min}$. Hearts were 'freeze-clamped' between aluminium tongs cooled in liquid nitrogen and pulverised under liquid $\mathrm{N}_{2}$ in a pestle and mortar. The powders were stored at $-80^{\circ} \mathrm{C}$.

\section{Cardiomyocyte cultures}

Neonatal rat ventricular myocytes were prepared and cultured from 3-5 d Sprague-Dawley rats (Charles River) as previously described [28]. Briefly, neonatal rats were culled by cervical dislocation, then the ventricles were dissected and dissociated by serial digestion at $37^{\circ} \mathrm{C}$ with $0.44 \mathrm{mg} / \mathrm{ml}(6800 \mathrm{U})$ Worthington Type II collagenase (supplied by Lonza) and $0.6 \mathrm{mg} / \mathrm{ml}$ pancreatin (Sigma-Aldrich, cat. No. P3292). Cell suspensions were recovered by centrifugation $(5 \mathrm{~min}, 60 \times \mathrm{g}$ ) and resuspended in plating medium (Dulbecco's modified Eagle's medium (DMEM)/medium 199 [4:1 (v/v)]) containing 15\% (v/v) foetal calf serum (FCS; Life Technologies) and $100 \mathrm{U} /$ $\mathrm{ml}$ penicillin and streptomycin. Cells were pre-plated on plastic tissue culture dishes $(30 \mathrm{~min})$ to remove noncardiomyocytes. Non-adherent cardiomyocytes were collected and viable cells counted by Trypan Blue (SigmaAldrich) exclusion using a haemocytometer. For immunoblotting or qPCR, viable cardiomyocytes were plated at a density of $4 \times 10^{6}$ cells/dish on $60 \mathrm{~mm}$ Primaria dishes pre-coated with sterile $1 \%$ (w/v) gelatin (SigmaAldrich). After $18 \mathrm{~h}$, myocytes were confluent and beating spontaneously. For immunostaining experiments, cardiomyocytes were plated at $1 \times 10^{6}$ cells/dish on $35 \mathrm{~mm}$ Primaria dishes containing glass coverslips pre-coated with sterile $1 \%(\mathrm{w} / \mathrm{v})$ gelatin followed by laminin $(20 \mu \mathrm{g} / \mathrm{ml}$ in PBS; Sigma-Aldrich). After $18 \mathrm{~h}$, myocytes were $\sim 50 \%$ confluent and beating spontaneously. For all experiments, the plating medium was withdrawn after $18 \mathrm{~h}$ and cells were incubated in serum-free maintenance medium (DMEM/medium 199 [4:1 (v/v)] containing $100 \mathrm{U} / \mathrm{ml}$ penicillin and streptomycin) for a further $24 \mathrm{~h}$ prior to experimentation.

\section{RNA preparation and qPCR}

Total RNA was prepared using RNA Bee (AMS Biotechnology Ltd) with $1 \mathrm{ml}$ per $4 \times 10^{6}$ cardiomyocytes or 10-15 mg mouse heart powder. RNA was prepared according to the manufacturer's instructions, dissolved in nuclease-free water and purity assessed from the $\mathrm{A}_{260} / \mathrm{A}_{280}$ measured using an Implen NanoPhotometer (values of 1.8-2.1 were considered acceptable). RNA concentrations were determined from the $A_{260}$ values. Quantitative PCR (qPCR) was performed as previously described [28]. Total RNA (1 $\mu \mathrm{g})$ was reverse transcribed to cDNA using High Capacity cDNA Reverse Transcription Kits with random primers (Applied Biosystems) according to the manufacturer's instructions. qPCR was performed using an ABI Real-Time PCR 7500 system (Applied Biosystems). Optical 96-well reaction plates were used with iTaq Universal SYBR Green Supermix (Bio-Rad Laboratories Inc.) according to the manufacturer's instructions. Primers were from PrimerDesign, Eurofins Genomics or Thermo Fisher Scientific (see Supplementary Table S4). GAPDH was used as the reference gene for the study. Results were normalised to GAPDH, and relative quantification was obtained using the $\Delta \mathrm{Ct}$ (threshold cycle) method; relative expression was calculated as $2^{-\Delta \Delta \mathrm{Ct}}$, and normalised to vehicle or time 0 .

\section{Immunoblotting}

Human heart samples were homogenised in RIPA buffer (Thermo Scientific, Cat. No. 89901) supplemented with Complete Mini, EDTA-free protease inhibitors (Roche, Cat. No. 11836170001) and PhosSTOP easypack phosphatase inhibitors (Roche, Cat. No. 04906837001). Samples were centrifuged $\left(3000 \times \mathbf{g}, 15 \mathrm{~min}, 4^{\circ} \mathrm{C}\right)$ and the supernatants collected. Protein quantification was performed using a Pierce BCA Protein Assay kit (Thermo Scientific, Cat. No. 23225). Samples were boiled with 0.33 vol SDS-polyacrylamide gel electrophoresis (SDS-PAGE) sample buffer [0.33 M Tris-HCl pH 6.8, 10\% (w/v) SDS, 13\% (v/v) glycerol, $133 \mathrm{mM}$ dithiothreitol, $0.2 \mathrm{mg} / \mathrm{ml}$ bromophenol blue]. 
Rodent hearts were ground to powder under liquid $\mathrm{N}_{2}$. Samples (15-20 mg) were extracted in 8 vol (relative to powder weight) Buffer A [20 mM $\beta$-glycerophosphate ( $\mathrm{pH} 7.5), 50 \mathrm{mM} \mathrm{NaF}, 2 \mathrm{mM}$ ethylenediamine tetraacetic acid (EDTA), 1\% (v/v) Triton X-100, $5 \mathrm{mM}$ dithiothreitol] containing protease and phosphatase inhibitors [10 mM benzamidine, $0.2 \mathrm{mM}$ leupeptin, $0.01 \mathrm{mM}$ trans-epoxy succinyl-l-leucylamido-(4-guanidino)butane, $0.3 \mathrm{mM}$ phenylmethylsulphonyl fluoride, $4 \mu \mathrm{M}$ microcystin]. For total cell extracts, cells were washed with ice-cold PBS and scraped into $150 \mu \mathrm{l}$ Buffer A containing protease and phosphatase inhibitors. Samples were vortexed and extracted on ice $(10 \mathrm{~min})$. Extracts were centrifuged $\left(10000 \times \mathrm{g}, 10 \mathrm{~min}, 4^{\circ} \mathrm{C}\right)$. The supernatants were removed, a sample was taken for protein assay and the remainder boiled with $0.33 \mathrm{vol}$ sample buffer. Protein concentrations were determined by Bio-Rad Bradford assay using bovine serum albumin (BSA) standards.

For analysis of cytosolic and nuclear extracts, cells were washed in ice-cold PBS and harvested into $150 \mu \mathrm{l}$ of hypotonic buffer (10 mM Hepes, $\mathrm{pH} 7.9,10 \mathrm{mM} \mathrm{KCl}, 1.5 \mathrm{mM} \mathrm{MgCl}_{2}, 0.3 \mathrm{mM} \mathrm{Na}_{3} \mathrm{VO}_{4}$ ) containing protease and phosphatase inhibitors. Samples were centrifuged $\left(10000 \times \mathrm{g}, 4^{\circ} \mathrm{C}, 5 \mathrm{~min}\right)$ and the supernatants (cytosolic fractions) boiled with $50 \mu \mathrm{l}$ of sample buffer. Pellets were resuspended in $50 \mu \mathrm{l}$ of nuclear extraction buffer $(20 \mathrm{mM}$ Hepes pH 7.9, $420 \mathrm{mM} \mathrm{NaCl}, 1.5 \mathrm{mM} \mathrm{MgCl} 2,0.2 \mathrm{mM}$ EDTA, 25\% (v/v) glycerol, $0.3 \mathrm{mM} \mathrm{Na} \mathrm{VO}_{4}$ ) containing protease and phosphatase inhibitors, and extracted on ice for $1 \mathrm{~h}$ with occasional vortex-mixing. The samples were centrifuged $\left(10000 \times \mathrm{g}, 4^{\circ} \mathrm{C}, 5 \mathrm{~min}\right)$ and the supernatant nuclear extracts boiled with $20 \mu \mathrm{l}$ sample buffer.

Proteins were separated by SDS-PAGE on $10 \%(\mathrm{w} / \mathrm{v})$ polyacrylamide resolving gels with $6 \%$ stacking gels, transferred electrophoretically to nitrocellulose using a Bio-Rad semi-dry transfer cell $(10 \mathrm{~V}, 60 \mathrm{~min})$ and detected as previously described [28]. Bands were visualised by enhanced chemiluminescence using ECL Prime Western Blotting detection reagents and using an ImageQuant LAS4000 system (GE Healthcare). ImageQuant TL 8.1 software (GE Healthcare) was used for densitometric analysis. Raw values for phosphorylated kinases were normalised to the total kinase. Values for all samples were normalised to the mean of the controls. Details of all primary and secondary antibodies are in Supplementary Table S5.

\section{Immunostaining}

Cardiomyocytes were washed with ice-cold PBS and fixed in 3.7\% (v/v) formaldehyde in PBS (10 min, room temperature). Cells were permeabilised with $0.3 \%$ (v/v) Triton X-100 (10 min, room temperature) in PBS, and non-specific binding blocked with $1 \%(\mathrm{w} / \mathrm{v}$ ) fatty acid-free BSA (Sigma-Aldrich, U.K.) in PBS containing $0.1 \%$ (v/v) Triton X-100 (10 min, room temperature). All incubations were at room temperature in a humidified chamber, and coverslips were washed three times in PBS after each stage of the immunostaining procedure. Cardiomyocytes were stained with mouse monoclonal primary antibodies to troponin $\mathrm{T}(60 \mathrm{~min})$ with detection using anti-mouse immunoglobulin secondary antibodies coupled to Alexa-Fluor 488 (60 min). Details of antibodies are in Supplementary Table S5. Coverslips were mounted using fluorescence mounting medium (Dako) and viewed with a Zeiss Axioskop fluorescence microscope using a $40 \times$ objective. Digital images were captured using a Canon PowerShot G3 camera using a 1.8 $\times$ digital zoom and the cell surface area of individual cardiomyocytes measured using ImageJ. The area was measured for all cells within at least three fields of view ( $>50$ cells) for each condition in each experiment and the mean values taken as a single observation. We conducted four or six experiments with independent cardiomyocyte preparations as indicated. Images were cropped for presentation using Adobe Photoshop CC.

\section{Fast protein liquid chromatography (FPLC), Raf kinase immunoprecipitation and protein kinase assays}

Protein complexes were separated according to relative molecular mass using an Äkta ${ }^{\mathrm{TM}}$ FPLC system with a Superdex 200 HR 10/30 column (GE Healthcare) and $24 \times 10^{6}$ cardiomyocytes per sample. Fractions (equal volumes) were immunoblotted as described above. The column was calibrated using gel filtration calibration kits (GE Healthcare). BRAF or RAF1 was immunoprecipitated and assayed with GST-MKK1 as substrate, largely as described in [29] using $1 \mu \mathrm{g}$ of antibody per sample and $4 \times 10^{6}$ NRVMs per sample. Samples of total extracts, immunoprecipitates and supernatants were boiled in sample buffer for immunoblotting with antibodies selective for phosphorylated or total MKK1/2, BRAF or RAF1.

\section{Statistical analysis}

Data analysis used Microsoft Excel and GraphPad Prism 8.0. Statistical analysis was performed using GraphPad Prism 8.0 with two-tailed unpaired $t$-tests, or two-tailed one-way or two-way ANOVA as indicated in the 
Figure Legends. A multiple comparison test was used in combination with ANOVA. A Grubb's outlier test was applied to the data, and outliers excluded from the analysis. Graphs were plotted with GraphPad Prism 8.0. Specific $P$ values are provided with significance levels of $P<0.05$ in bold type.

\section{Results}

\section{BRAF is up-regulated in human failing hearts}

BRAF and RAF1 (but not ARAF) mRNA (Figure 1A) and protein (Figure 1B,C) were significantly up-regulated in samples from a cohort of 12 patients with heart failure of mixed non-ischaemic aetiology compared with normal controls. Since this group is relatively small, we mined an RNASeq database of patients with welldefined dilated cardiomyopathy $(n=97) v s$ normal controls $(n=108)$ [22]. ARAF, BRAF and RAF1 transcripts were readily detected in human hearts, but only $B R A F$ transcript expression was significantly increased in
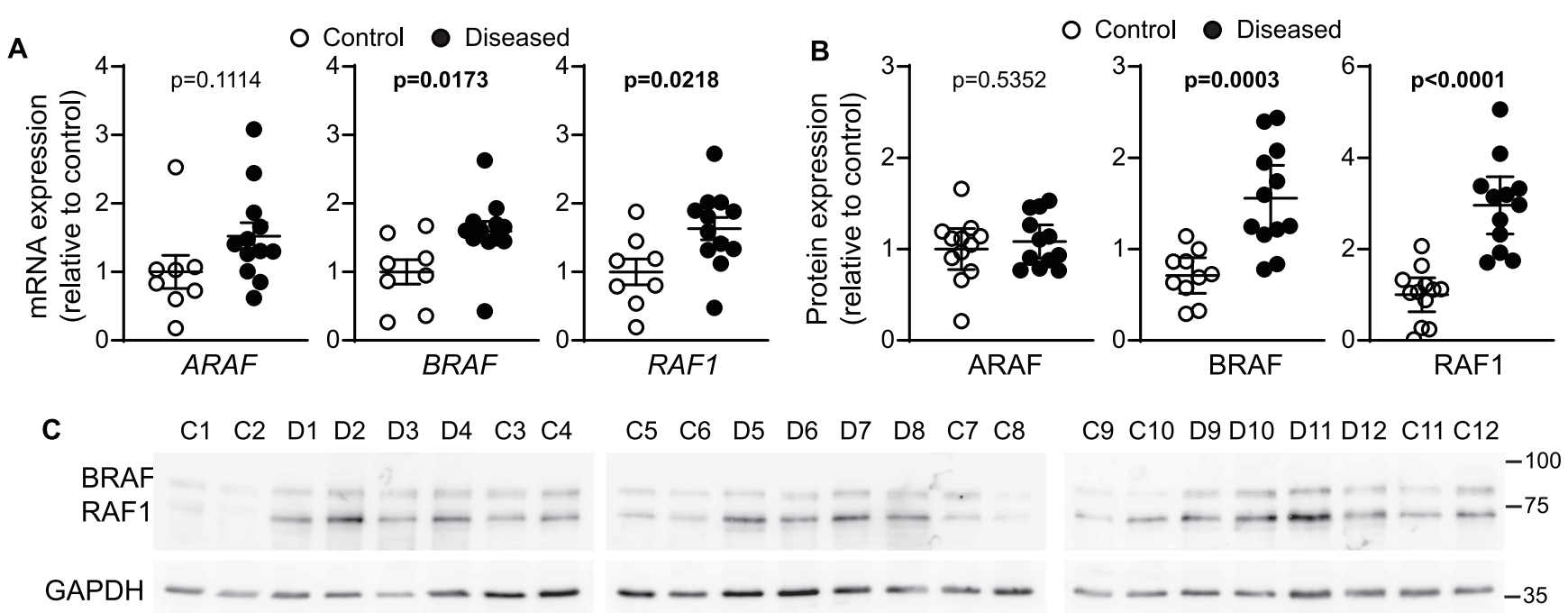

C9 C10 D9 D10 D11 D12 C11 C12

BRAF

RAF1

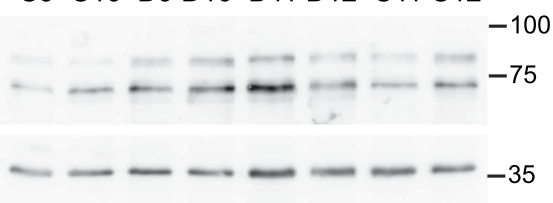

$-100$

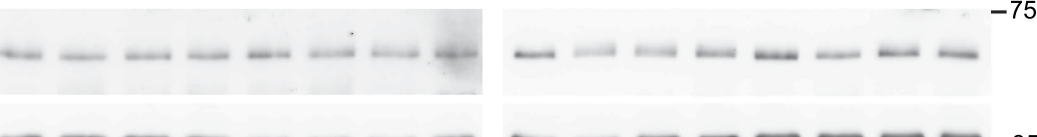

GAPDH

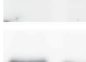

D

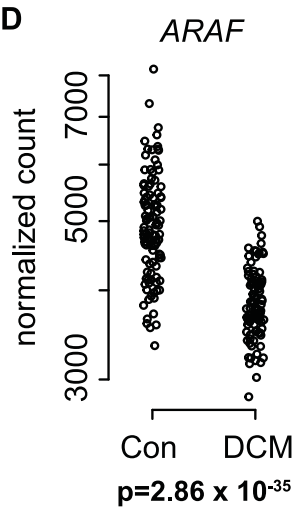

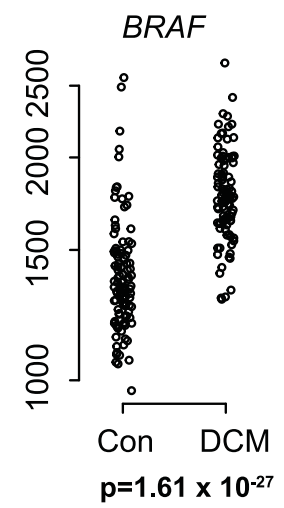

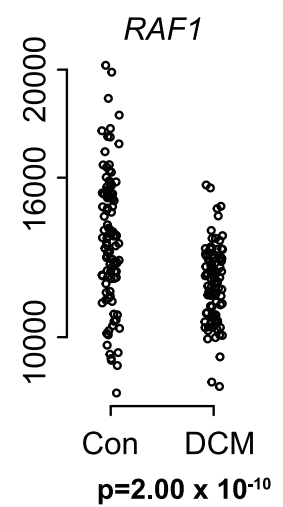

Figure 1. BRAF is up-regulated in human failing hearts.

(A-C) Expression of ARAF, BRAF and RAF1 in human heart samples showed increased expression of BRAF and RAF1 in failing hearts compared with controls (unpaired two-tailed $t$-tests). (A) mRNA expression was assessed by qPCR. (B and C) Proteins were analysed by immunoblotting. Blots were reprobed for GAPDH. Densitometric analysis of the blots $(\mathbf{C})$ is in $(\mathbf{B})$. Positions of relative molecular mass markers $(\mathrm{kDa})$ are on the right of the immunoblots. Data were normalised to GAPDH and expressed relative to the mean of the controls. (D) Data were mined from an RNASeq database of patients with dilated cardiomyopathy $(n=97)$ and normal controls $(n=108)$. Data for individual samples are shown with adjusted $P$ values. 
dilated cardiomyopathy samples; ARAF and RAF1 transcripts showed decreased expression (Figure 1D). Thus, BRAF is potentially important in human heart failure.

\section{Activation of cardiomyocyte BRAF promotes hypertrophy}

To assess the role of BRAF in cardiomyocytes, we used a genetic model with tamoxifen-inducible Cre regulated by a Myh6 promoter for cardiomyocyte-specific expression $\left(\mathrm{Cre}^{\mathrm{MCM}}\right)$ [25]. This allowed selective manipulation of BRAF activity in adult cardiomyocytes, avoiding complications during development. BRAF was activated in cardiomyocytes using a floxed gene for $\mathrm{BRAF}^{\mathrm{V} 600 \mathrm{E}}$ knock-in into the endogenous gene [24]. We used mice heterozygous for the floxed gene for $\mathrm{BRAF}^{\mathrm{V} 600 \mathrm{E}}$ knock-in and hemizygous for Cre (i.e. $\mathrm{BRAF}^{\mathrm{V} 600 \mathrm{E} / \mathrm{WT}} / \mathrm{Cre}^{\mathrm{MCM} /-}$ ). Following baseline echocardiography, recombination was induced with a single injection of $40 \mathrm{mg} / \mathrm{kg}$ tamoxifen, a dose established to induce recombination in the absence of significant cardiomyopathy [30], and an approach which allows rapid clearance from the body (within 24-72 h) [31]. Echocardiograms were taken and mice killed at intervals as shown in the scheme in Figure 2A. Tamoxifen-treatment induced selective recombination in the heart (Figure 2B). Recombination in heart tissue is not complete because $\sim 70 \%$ of cells in the heart are cardiac non-myocytes (e.g. endothelial cells and fibroblasts). At $24 \mathrm{~h}$, there was no change in expression of BRAF mRNA expression, although there was a small, significant increase and decrease in ARAF and RAF1 mRNA expression, respectively (Figure 2C). This had normalised by $10 \mathrm{~d}$, with no significant effects on expression of any of the RAF kinases at the mRNA or protein level (Figure 2C,D).

$\mathrm{BRAF}^{\mathrm{V} 600 \mathrm{E}}$ knock-in increased phosphorylation (i.e. activation) of MKK1/2 over $10 \mathrm{~d}$ (Figure 2D). Although we could not detect an increase in phosphorylation of $\mathrm{ERK} 1 / 2, \mathrm{BRAF}^{\mathrm{V} 600 \mathrm{E}}$ knock-in induced some increase phosphorylation of p90 ribosomal S6 kinase (p90RSK) (Figure 2E; see Discussion), a well-established ERK1/2 substrate [32]. ERK1/2 can promote phosphorylation of p70 ribosomal S6 kinase (p70S6K) [33], but there was no increase in phosphorylation of key sites (S371/S389) associated with p70S6K activation with BRAF ${ }^{\mathrm{V} 600 \mathrm{E}}$ knock-in. p70S6K activation is more generally associated with activation of Akt with phosphorylation of Akt (T308) in the context of mTOR complex 1 (mTORC1), a key driver of protein synthesis and inhibitor of autophagy [34]. We did not detect any increase in phosphorylation of Akt(T308), but we did detect an increase in phosphorylation of $\mathrm{Akt}(\mathrm{S} 473)$, a site associated with activation of Akt in the context of mTORC2 and the regulation of gene expression via Forkhead transcription factors [34]. Consistent with activation of the ERK1/2 cascade, $\mathrm{BRAF}^{\mathrm{V} 600 \mathrm{E}}$ knock-in promoted an increase in expression of mRNAs for ERK1/2-dependent immediate early genes associated with cardiac hypertrophy (e.g. Egr1, Dusp5, Fos, Jun and Myc) [28,35,36], along with hypertrophic gene markers ( $N p p a, N p p b, M y h 7$ ) [2], consistent with cardiac hypertrophy (Figure 2F,G). There was also a small, but significant increase in IL11 mRNA expression (known to drive ERK1/2-dependent fibrosis in the heart [37]) that, together with Collal and Lox, suggested there could be some fibrotic response. A small, but significant increase in $I L 1 \beta$ was detected suggesting there could be some inflammation. Tamoxifen alone induced a small increase in mRNA expression of $M y h 7$ in hearts of mice hemizygous for Cre only, but did not affect the other genes (Supplementary Table S6).

We assessed the effects of activated cardiomyocyte BRAF on cardiac function and dimensions using echocardiography (Figure 3; Supplementary Table S7). Assessment of left ventricular dimensions from M-mode shortaxis images of the heart indicated that activation of BRAF in cardiomyocytes increased diastolic and systolic wall thicknesses within $10 \mathrm{~d}$ of tamoxifen treatment (Figure 3A,B). This was associated with an increase in cardiomyocyte cross-sectional area indicative of hypertrophy (Figure 3C). Tamoxifen treatment of mice hemizygous for Cre did not have any significant effect on cardiac function or dimensions (Supplementary Table S8). The increase in left ventricular wall thickness was sustained over 6 weeks (Figure 3D,E). By this stage, there was a significant decrease in internal diameters, particular in systole, suggesting there was likely to be a change in cardiac function. Assessment of B-mode long-axis images of the heart at 6 weeks with strain analysis using speckle-tracking software demonstrated that activation of cardiomyocyte BRAF increased the predicted LV mass, with a reduction in diastolic and systolic volumes, increasing ejection fraction and fractional shortening (Figure 3F; Supplementary Table S9). Histological assessment demonstrated that the increase in cardiomyocyte cross-sectional was sustained after 6 weeks of BRAF activation (Figure 3G). Despite the increase in Nppa, Nppb, IL11, Col1a1, Lox and IL1 $\beta$ mRNAs (Figure 2E,F), there was no evidence for any increase in cardiac fibrosis or cellular damage at $10 \mathrm{~d}$ or 6 weeks (Figure 3C,G). We conclude that the changes in mRNA expression of these genes were either too small to elicit a significant effect overall, or that these genes were subject to translational regulation. Overall, the data indicate that activation of endogenous cardiomyocyte BRAF induces cardiac hypertrophy and enhances cardiac function. 


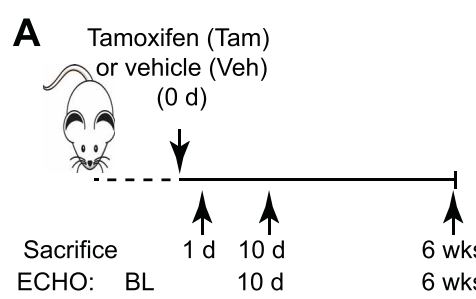

B

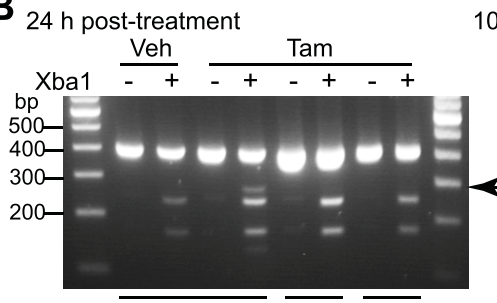

D
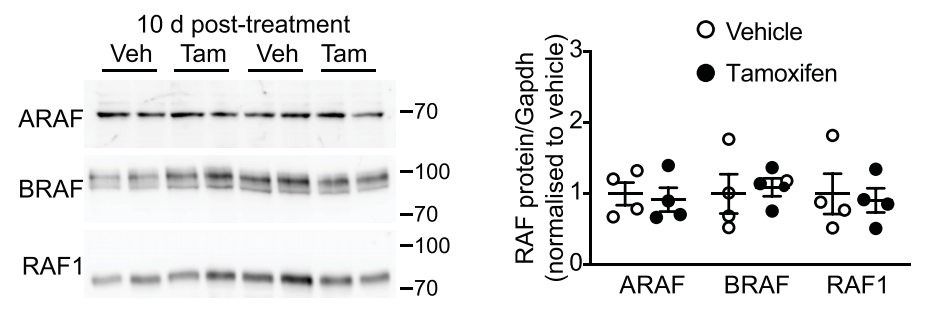

Phospho-MKK1/2 $=-\infty=-50$
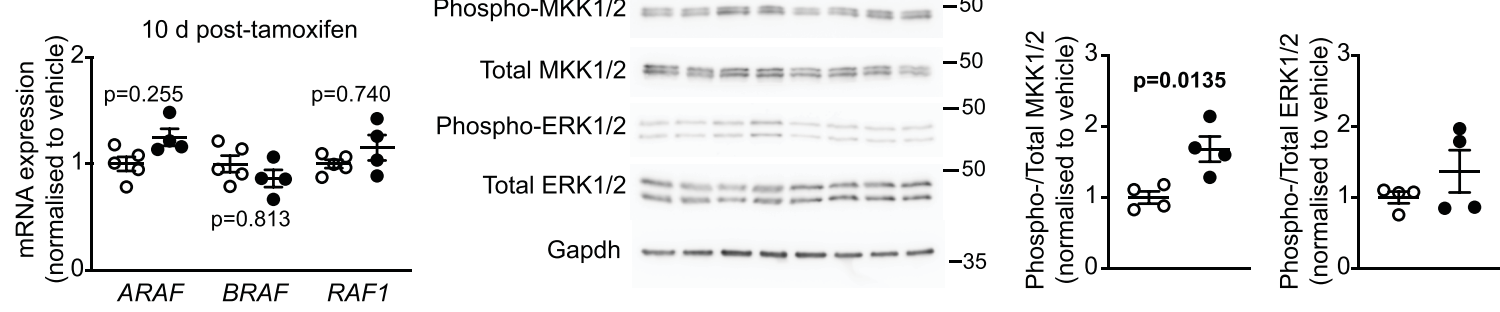

E

$\underline{\text { Veh Tam }} \underline{\text { Veh }} \stackrel{\text { Tam }}{\text { Veh }}$

Phosphop90RSK(S380/573)

O Vehicle Total p90RSK

- Tamoxifen
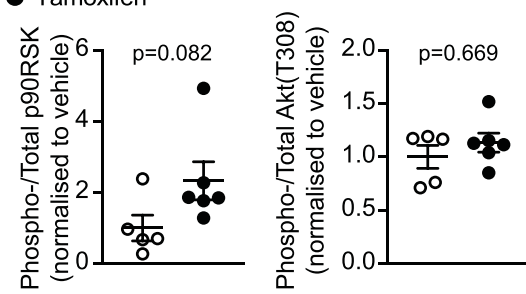

$\mathbf{G}$

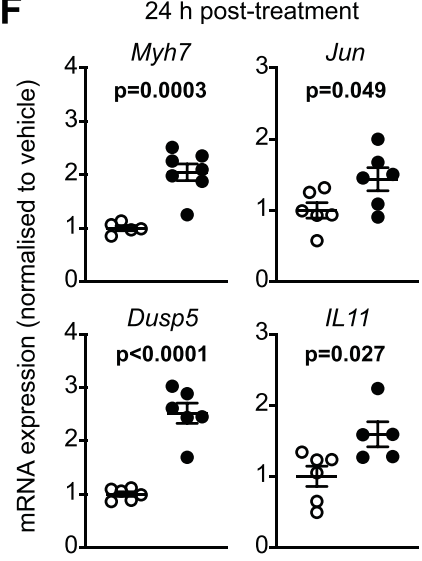

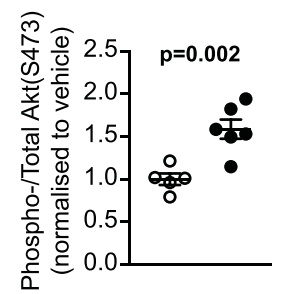

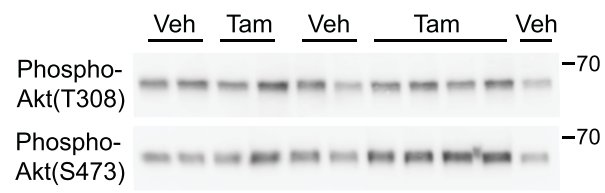

Total Akt $-\cdots-\cdots----70$

Phospho-

p70s6K

(S371/389)

Total p70S6K $=-\cdots--0-\cdots--70$

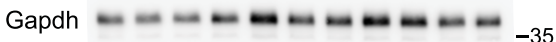

$10 \mathrm{~d}$ post-treatment

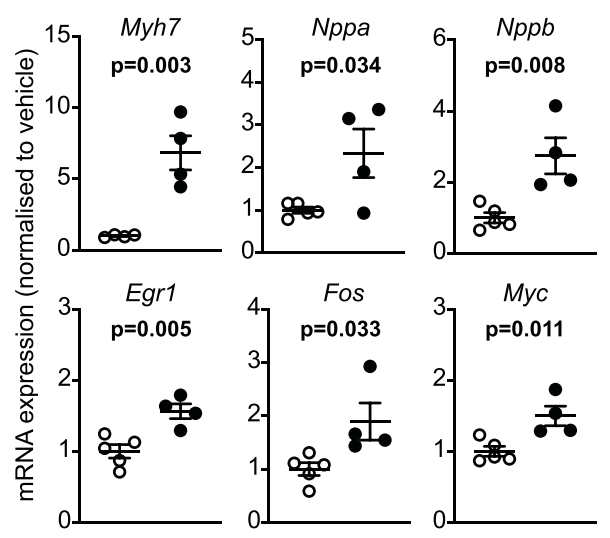

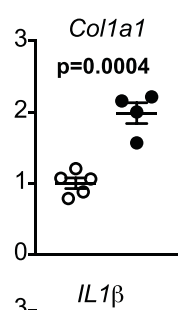
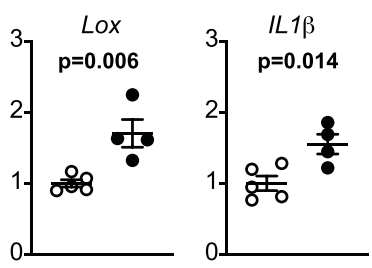

Figure 2. Activated cardiomyocyte BRAF induces changes in cardiac gene expression in mouse hearts in vivo. Part 1 of 2 (A) Study protocol. Mice heterozygous for floxed BRAF ${ }^{\mathrm{V} 600 \mathrm{E}}$ and hemizygous for Myh6-directed tamoxifen-inducible Cre were treated with $40 \mathrm{mg} / \mathrm{kg}$ (i.p.) tamoxifen (Tam) or vehicle (Veh). Echocardiograms (ECHO) were taken at baseline (BL) then at $10 \mathrm{~d}$ or 
Figure 2. Activated cardiomyocyte BRAF induces changes in cardiac gene expression in mouse hearts in vivo. Part 2 of 2 6 weeks after tamoxifen treatment. (B) Confirmation of recombination for BRAF ${ }^{\mathrm{V} 600 \mathrm{E}}$ knock-in was based on introduction of an Xba1 restriction site in the mRNA following recombination. cDNA was prepared from RNA extracted from heart, liver or kidney, $24 \mathrm{~h}$ or $10 \mathrm{~d}$ after treatment with tamoxifen or vehicle. cDNA was PCR amplified and subject to Xba1 digestion, resulting in appearance of additional bands (arrows) in hearts (not liver or kidney) following treatment with tamoxifen, but not vehicle. (C) qPCR analysis of mRNA expression of RAF isoforms in samples of mouse hearts treated with vehicle or tamoxifen for $24 \mathrm{~h}$ (upper panel) or $10 \mathrm{~d}$ (lower panel). (D and E) Immunoblotting of RAF isoforms or Gapdh, in addition to phosphorylated (phospho-) or total MKK1/2, ERK1/2, p90RSK, Akt and p70S6K in samples of mouse hearts treated with vehicle or tamoxifen. Representative immunoblots are shown (positions of relative molecular mass markers in $\mathrm{kDa}$ are on the right of each blot) with densitometric analysis. ( $\mathbf{F}$ and $\mathbf{G})$ qPCR for mRNA expression in mouse hearts after tamoxifen-treatment for $24 \mathrm{~h}(\mathbf{F})$ or $10 \mathrm{~d}(\mathbf{G})$. Data are provided as individual values with mean \pm SEM. Statistical analysis: unpaired two-tailed $t$-test.

\section{BRAF and RAF1 form high molecular mass complexes in cardiomyocytes and are activated by the Type 1 RAF inhibitor, SB590885}

ARAF, RAF1 and BRAF have predicted relative molecular masses of 68,73 and $85 \mathrm{kDa}$, but RAF1/BRAF operate as dimers to activate MKK1/2 [20], potentially in a multiprotein complex, and ARAF may serve as a scaffold [38]. In proliferating cells, activation of RAF signalling by 'RAF paradox'-inducing compounds may be associated with dimer formation or stabilisation [20]. However, cardiomyocytes are terminally differentiated and RAF signalling is rapid in these cells (e.g. activation of RAF1 by the pro-hypertrophic agonist endothelin-1 is maximal within 1-3 min [11]), suggesting the complexes may be pre-formed. To explore this further, we used cardiomyocytes isolated from neonatal rats at post-natal day 3-5 when they withdraw from the cell cycle [39]. Cells were cultured on a rigid surface that reduces proliferative potential even of cardiomyocytes isolated from rats at post-natal day 1 [40]. We separated RAF complexes from cardiomyocytes using Superdex 200 gel filtration FPLC (Figure 4A-D). BRAF was detected largely in fractions 34-38 correlating with relative molecular mass complexes of $230-370 \mathrm{kDa}$, whereas RAF1 and ARAF eluted across a wider range corresponding to relative molecular mass complexes of $260-580 \mathrm{kDa}$. We detected little RAF in fractions $40 / 41$ or $46-48$ where dimers or monomers would be expected to elute, suggesting that RAFs predominantly exist in multi-protein complexes in cardiomyocytes. To assess BRAF and RAF1 activities, we first immunoblotted cardiomyocyte extracts with antibodies to the activating phosphorylation sites (Figure 4E). As shown previously [11], endothelin-1 substantially increased phosphorylation of RAF1(S338). This was associated with a band shift (appearance of bands of apparently higher molecular mass) of total RAF1 protein consistent with phosphorylation. Endothelin-1 had little effect on phosphorylation of BRAF(S445), but induced a band shift of the total protein suggesting that there was or had been activation. Using in vitro protein immunokinase assays, we determined that activation of RAF1 by endothelin-1 was substantially greater than BRAF (Figure 4F). RAF1 was detected in BRAF immunoprecipitates, but it was difficult to detect BRAF in RAF1 immunoprecipitates. This may indicate that RAF1 is of higher abundance and interacts with proteins other than BRAF or it could be a technical issue relating to the affinities of the different antibodies. Nevertheless, BRAF and RAF1 co-immunoprecipitated and activation by endothelin- 1 did not affect the degree of association (Figure 4F).

We next assessed the effects of SB590885, a Type 1 RAF kinase inhibitor with 11-fold higher affinity for BRAF than RAF1 [41], on activation of ERK1/2 in cardiomyocytes. As expected, high concentrations $(10 \mu \mathrm{M})$ inhibited ERK1/2 phosphorylation, but concentrations of $0.1-0.01 \mu \mathrm{M}$ SB590885 activated ERK1/2 (Figure 4G). Low concentrations of SB590885 also increased MKK1/2-activating activity in RAF1 or BRAF immunoprecipitates (Figure 4F). We assessed the time courses for ERK1/2 activation by SB590885 in cardiomyocytes, comparing $1 \mu \mathrm{M}$ (with no effect on ERK1/2 phosphorylation at $20 \mathrm{~min}$ ) with $0.1 \mu \mathrm{M}$ (that activated ERK1/2). The higher concentration activated ERK1/2 at later times giving a similar degree of activation at $1 \mathrm{~h}$ as $0.1 \mu \mathrm{M}$, and ERK1/2 activation remained elevated over at least $8 \mathrm{~h}$ (Figure $4 \mathrm{H}, \mathrm{I}$ ). This may reflect reducing concentrations of active, available compound as it is metabolised. To confirm that the RAF paradox was relevant for the adult heart, we assessed the effects of SB590885 on ERK1/2 phosphorylation in Langendorff-perfused adult rat hearts. Consistent with cardiomyocytes, SB590885 activated ERK1/2 (Figure 4J).

In cardiomyocytes, hypertrophic agonists (e.g. endothelin-1) increase activated ERK1/2 in the nuclear compartment without net accumulation of total ERK1/2 and this regulates gene expression [36]. SB590885 increased ERK1/2 phosphorylation in the nuclear-enriched protein fraction (Figure 5A,B) and increased 
A
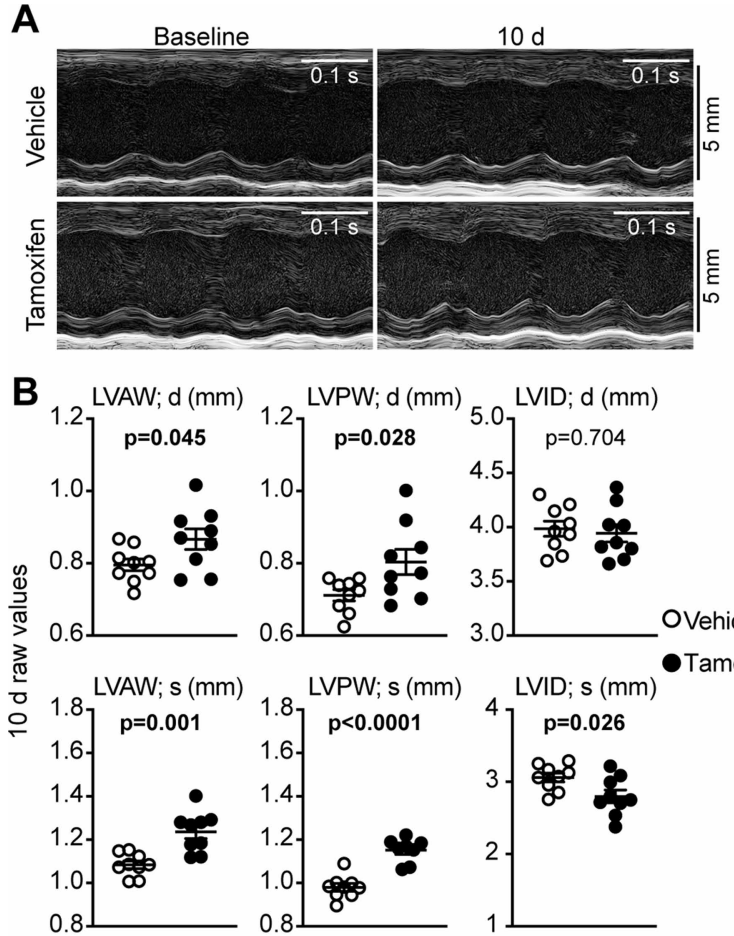

D

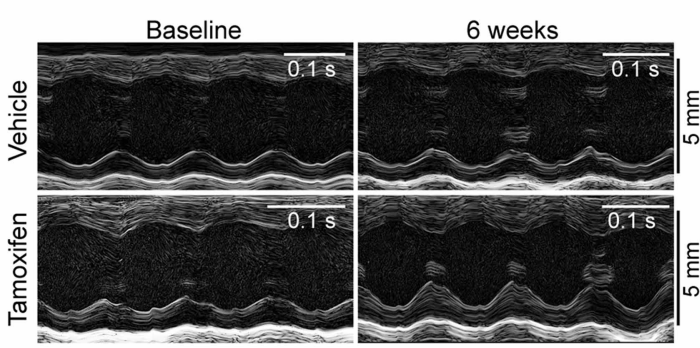

E

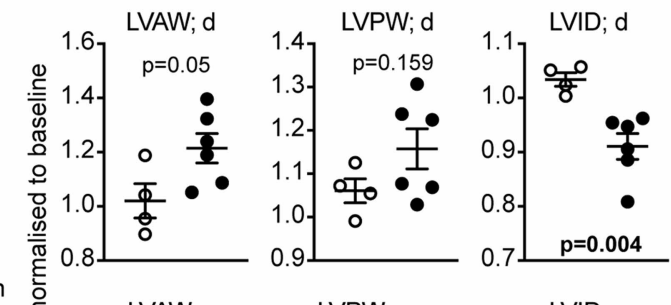

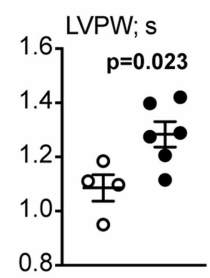

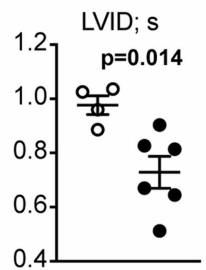

C
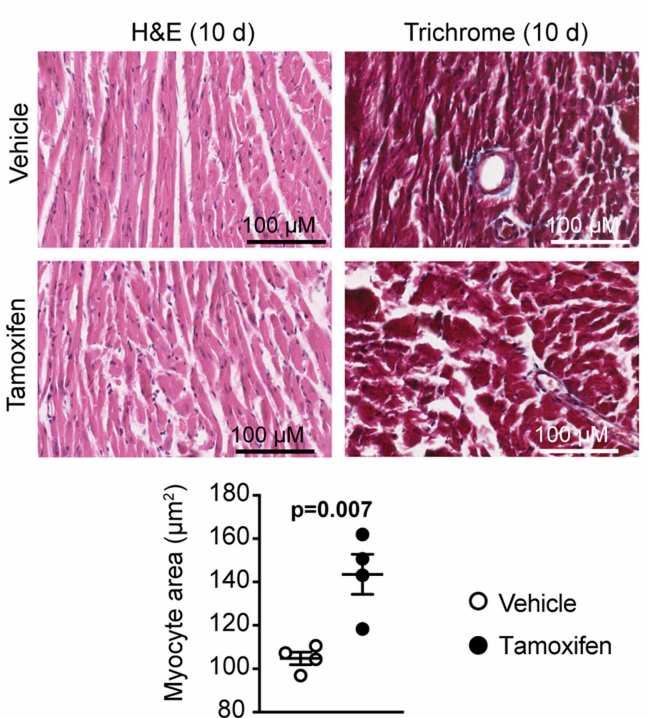

$\mathbf{F}$
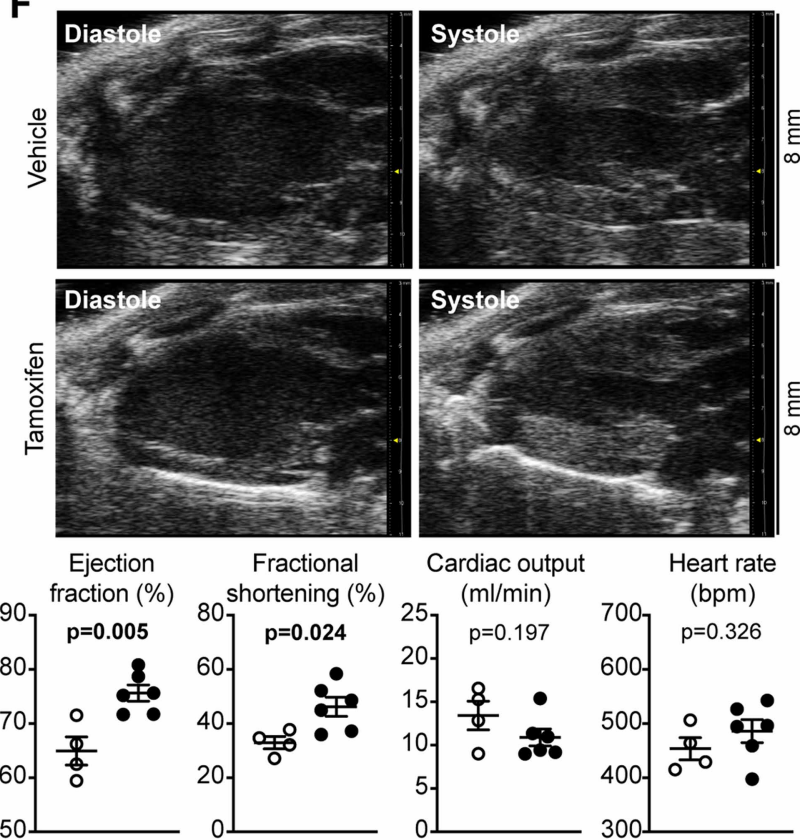

Cardiac output Heart rate

O Vehicle

- Tamoxifen

G

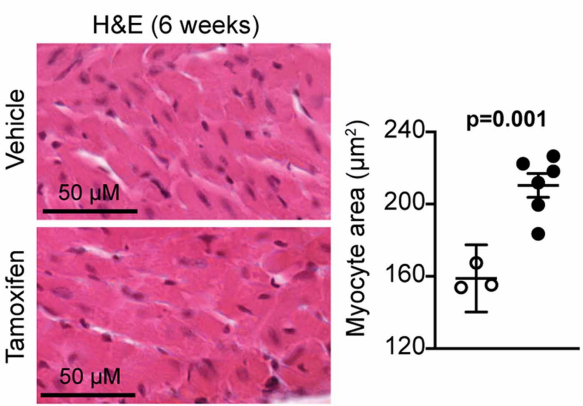

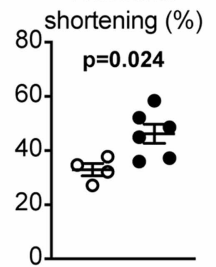

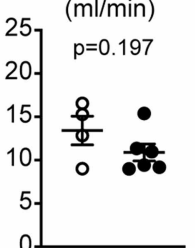

40

(bpm)

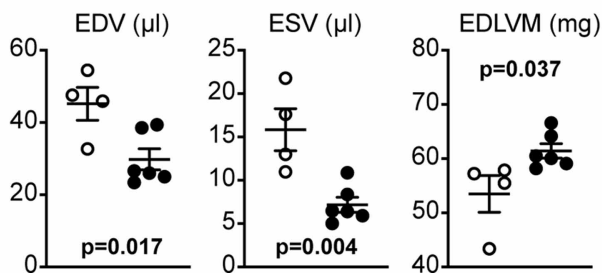

Figure 3. Activated cardiomyocyte BRAF promotes cardiac hypertrophy in mouse hearts in vivo. Mice heterozygous for floxed BRAF ${ }^{\mathrm{V} 600 \mathrm{E}}$ and hemizygous for Myh6-directed tamoxifen-inducible Cre (BRAF ${ }^{\mathrm{V} 600 \mathrm{E} / \mathrm{WT}} / \mathrm{Cre}^{\mathrm{MCM} /-}$ ) were treated with a single dose of $40 \mathrm{mg} / \mathrm{kg}$ (i.p.) tamoxifen or vehicle, and maintained for $10 \mathrm{~d}$ (A-C) or 6 weeks (D-G). 

short-axis views for individual mice before and after treatment. (B and E) Quantification of echocardiograms represented in panels $\mathbf{A}$ and $\mathbf{D}$, respectively. LV, left ventricle; AW, anterior wall; PW, posterior wall; ID, internal diameter; d, diastole; s, systole. C and G, Sections of BRAF ${ }^{\mathrm{V} 600 \mathrm{E} / \mathrm{WT}} / \mathrm{Cre}^{\mathrm{MCM} /-}$ mouse hearts stained with haemotoxylin and eosin (H\&E) or Masson's Trichrome were analysed. Representative images are shown with quantification provided below (C) or in the right panel (G). (F) B-mode images of long-axis views of the heart were used for speckle-tracking strain analysis. Representative images are in the upper panels with cardiac function data in the lower panels. EDV, end diastolic volume; ESV, end systolic volume; EDLVM, end diastolic left ventricular mass; ESLVM, end systolic LV mass. Data are provided as individual points with means \pm SEM. Statistical analysis: unpaired two-tailed $t$-test.

expression of a range of immediate early genes (Figure 5C). The latter was inhibited by the MKK1/2 inhibitor PD184352 $(2 \mu \mathrm{M})$ [42], confirming the response was a direct consequence of ERK1/2 signalling. We next assessed whether SB590885 promoted cardiomyocyte hypertrophy, using planimetry to measure the cell surface area of individual cardiomyocytes. Consistent with a role for ERK1/2 signalling in cardiomyocyte hypertrophy, SB590885 increased cardiomyocyte area (Figure 5D).

SB590885 was developed as a potential therapeutic agent but is not used clinically. Our data suggest it may not be particularly stable even in cells in culture (Figure $4 \mathrm{H}, \mathrm{I}$ ) so we considered if other RAF inhibitors may exert a similar effect. Of the inhibitors in clinical use, encorafenib is available as an anti-cancer therapy for use in combination with the MKK1/2 inhibitor binimetinib [43]. It is not clear if encorafenib is a Type 1 RAF inhibitor, but it activated ERK1/2 signalling in cultured cardiomyocytes at low concentrations $(0.001-1 \mu \mathrm{M})$ (Figure 5E), and promoted an increase in cardiomyocyte cell surface area (Figure 5D). To determine if Raf inhibitors that promote Raf paradox signalling can synergise with other pro-hypertrophic stimuli that activate ERK1/2, we assessed the concentration-dependent effects of SB590885 or encorafenib on activation of ERK1/2 by A61603, an $\alpha_{1 \mathrm{~A}}$-adrenergic receptor agonist that promotes a relatively modest increase in ERK1/2 phosphorylation [36]. Both drugs inhibited phosphorylation of MKK1/2 and ERK1/2 by A61603 at the highest concentrations assessed, with a suggestion that there might be some signal enhancement at lower concentrations (Figure 5F). It is possible that the effects of drugs that promote Raf paradox signalling can synergise with other hypertrophic stimuli, but this will presumably depend on the degree of stimulation of the pathway and the specific stimulus.

\section{SB590885 and encorafenib promote cardiomyocyte and cardiac hypertrophy in vivo}

Our studies of SB590885 and encorafenib in cardiomyocytes suggested they should be pro-hypertrophic in vivo. To determine if this is the case, we treated adult male mice with SB590885 using osmotic minipumps for drug delivery. We used echocardiography to assess the effects on cardiac dimensions (from M-mode images of short-axis views of the heart) and function (by strain analysis of B-mode images of the long-axis views of the heart). As mentioned above, SB590885 is not used clinically, and so we selected a concentration based on the dose of the alternative RAF inhibitor, dabrafenib. Dabrafenib is administered orally in humans at up to $150 \mathrm{mg}$, twice daily (i.e. $\sim 3-5 \mathrm{mg} / \mathrm{kg} / \mathrm{d}$ ). $\mathrm{IC}_{50}$ values for inhibition of BRAF and RAF1 by dabrafenib are $5.2 \mathrm{nM}$ and $6.3 \mathrm{nM}$, respectively [19]. $\mathrm{IC}_{50}$ values for SB590885 for inhibition of BRAF and RAF1 are $\sim 6$-fold less than dabrafenib for inhibition of RAF1 [41], so we used $0.5 \mathrm{mg} / \mathrm{kg} / \mathrm{d}$. SB590885 promoted a small increase in diastolic and systolic left ventricular wall thickness within $3 \mathrm{~d}$ (Figure 6A; Supplementary Table S10). This was associated with a significant increase in predicted left ventricular mass (Figure 6B; Supplementary Table S9). Consistent with the echocardiography, SB590885 induced an increase in cardiomyocyte cross-sectional area without any overt increase in fibrosis or infiltrating cells (Figure 6C). However, in contrast with BRAF ${ }^{\mathrm{V} 600 \mathrm{E}}$ knock-in (Figure 2E,F), there were no significant changes in mRNA expression of $N p p a$ or $N p p b$ (Figure 6D).

We next assessed the effects of encorafenib on mouse hearts in vivo. Encorafenib is used at $300-450 \mathrm{mg} / \mathrm{d}$ in humans, so we selected a dose of $3 \mathrm{mg} / \mathrm{kg} / \mathrm{d}$ for delivery by osmotic minipumps. Like SB590885, encorafenib increased systolic/diastolic anterior and posterior wall thickness (Figure 6E; Supplementary Table S10) and left ventricular mass (Figure 6F). Encorafenib also increased cardiomyocyte cross-sectional area (Figure 6G), consistent with cardiomyocyte hypertrophy without any increase fibrosis, and it did not affect expression of Nppa 
A
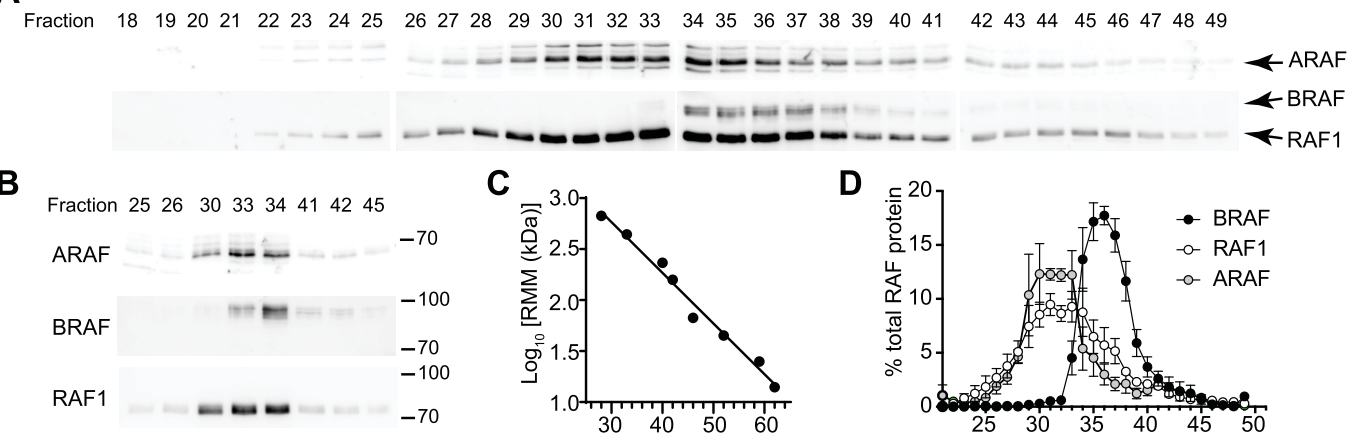

E

C

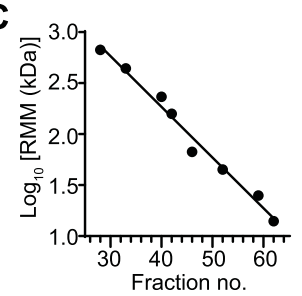

D.

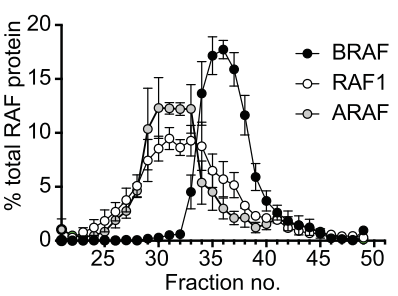

F

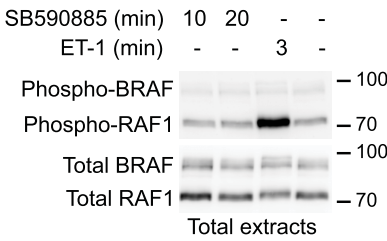

G

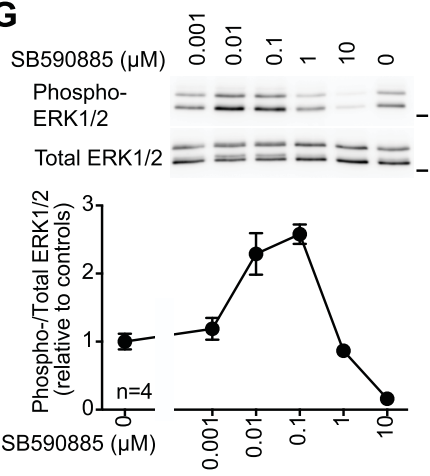

$\mathrm{SB} 590885(\mathrm{~min}) 1020$ - 101020 -

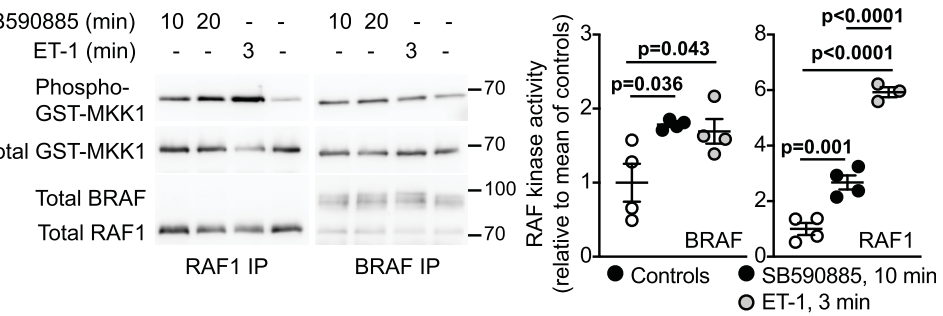

H

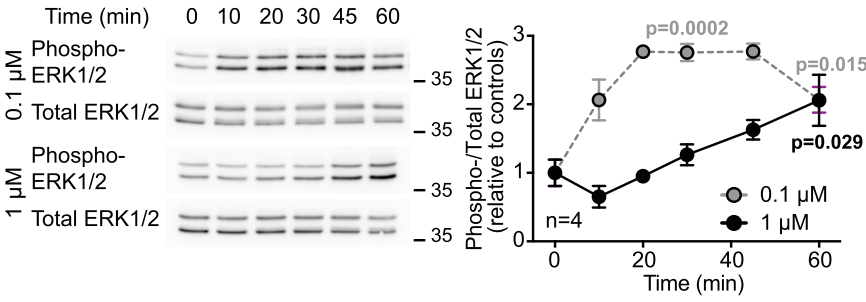

I Time (h) $0 \begin{array}{llllllll}0 & 0.3 & 0.7 & 1 & 2 & 4 & 6 & 8\end{array}$ Phospho-
ERK1/2 Total ERK1/2 ニニニニニニニニ

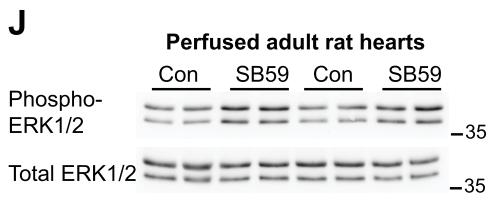

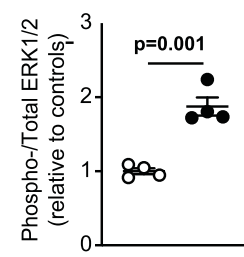

O Controls

- SB590885

Figure 4. Activation of RAF and ERK1/2 signalling in cardiomyocytes by the type 1 RAF kinase inhibitor, SB590885. Part 1 of 2 (A-D) Superdex 200 FPLC separation of RAF complexes in unstimulated neonatal rat cardiomyocytes. Fractions were immunoblotted with antibodies to ARAF, or BRAF and RAF1. Representative blots of all column fractions (A) and comparing expression in fractions across different blots (B) are shown. (C) Column calibration with relative molecular mass (RMM) standards. (D) Elution profiles of ARAF, BRAF and RAF1. Results are means \pm SEM $(n=3)$. (E) Immunoblots of extracts from neonatal rat cardiomyocytes exposed to $0.1 \mu \mathrm{M}$ SB590885 or $0.1 \mu \mathrm{M}$ endothelin-1 (ET-1) for the times indicated. Total extracts were immunoblotted for phosphorylated BRAF(S445) and RAF1(S338) (upper blot) or the total proteins (lower blot). Representative blots of four independent cardiomyocyte preparations are shown. (F) RAF kinase activities in neonatal rat cardiomyocytes exposed to the indicated concentrations of SB590885 or $0.1 \mu \mathrm{M}$ ET-1 for the times shown. Activities were assayed using GST-MKK1 following immunoprecipitation (IP) of RAF1 or BRAF. Phosphorylation of GST-MKK1 was detected with antibodies to phosphorylated MKK1/2. Assay samples were also immunoblotted for total MKK1/2, BRAF and RAF1. Representative blots are shown (left panels) with densitometric quantification (right panels; results are the ratio of phosphorylated/total GST-MKK1, normalised to the mean of controls). Data are provided for each experiment with means \pm SEM $(n=4)$. Statistical analysis used one-way ANOVA with Holm-Sidak's post-test. (G-J) Immunoblot analysis of phosphorylated (phospho-) and total ERK1/2 in extracts from neonatal rat cardiomyocytes (G,H and I) or Langendorff 
Figure 4. Activation of RAF and ERK1/2 signalling in cardiomyocytes by the type 1 RAF kinase inhibitor, SB590885. Part 2 of 2 perfused adult rat hearts $(\mathbf{J})$. Cells were exposed to the indicated concentrations of SB590885 for $20 \mathrm{~min}(\mathbf{G})$ or the indicated concentration of SB590885 for the times shown (H and $\mathbf{I})$. Rat hearts were perfused (15 min) under control conditions or with $1 \mu \mathrm{M}$ SB590885 (SB59) (J). Representative immunoblots are shown with densitometric analysis. Results of the cell experiments are provided as means \pm SEM for $n=3$ or 4 independent cell preparations as indicated. Heart perfusion data are provided as individual results with means \pm SEM $(n=4)$. Statistical analysis used one-way ANOVA with Holm-Sidak's post-test and individual $P$ values are provided. Positions of relative molecular mass markers $(\mathrm{kDa})$ are on the right of the immunoblots.

or Nppb mRNAs (Figure 6H). We conclude that Type 1 RAF kinase inhibitors promote cardiomyocyte hypertrophy in vivo, consistent with their effects in cultured cardiomyocytes. In vivo, the drugs increase cardiomyocyte cross-sectional area, in the absence of pathological features of cardiac hypertrophy such as fibrosis or inflammation.

Since SB590885 and encorafenib promoted an apparently physiological form of hypertrophy, it is possible that drugs such as these may be therapeutically useful for heart failure. To explore this, we conducted a limited study of the effects of SB590885 $(0.5 \mathrm{mg} / \mathrm{kg} / \mathrm{d})$ on the early phase of hypertension-induced cardiac hypertrophy induced by $0.8 \mathrm{mg} / \mathrm{kg} / \mathrm{d}$ angiotensin II (AngII) in mice over $7 \mathrm{~d}$. Cardiac function/dimensions were assessed by echocardiography using speckle-tracking analysis of B-mode long axis images, and hearts were taken for biochemical analysis. At $7 \mathrm{~d}$, AngII had no significant effects on heart rate or ejection fraction, but caused a decrease in cardiac output and stroke volume, presumably a result of increased left ventricular (LV) mass (i.e. hypertrophy) coupled with decreased end diastolic and systolic LV volume (Figure 7A). The data are consistent with diastolic dysfunction as reported for this model [44]. SB590885 did not affect heart rate or ejection fraction but appeared to have a small limiting effect on the reduction in cardiac output and stroke volume. This did not reflect a significant impact on the increase in LV mass, but there was a limitation in the reduction in end diastolic and systolic LV volume. To gain insight into the possible mechanisms, we assessed the effects on changes in gene expression induced by AngII. SB590885 had a modest effect to inhibit the increase in the hypertrophic gene markers $N p p a, N p p b$ and $M y h 7$ induced by AngII, and significantly suppressed the reduction in expression of Myh6 (Figure 7B). However, there was a much more pronounced and significant inhibition of AngII-induced up-regulation of markers of fibrosis (Ddr2, Colla1, Fn1, Postn; Figure 7C) and inflammation (IL1b, IL6; Figure 7D). Overall, the data suggest that SB590885 may improve cardiac function by reducing fibrosis and inflammation.

\section{Discussion}

BRAF signalling has been studied extensively in relation to cancer because of its oncogenic potential, but the role of BRAF in the heart has remained relatively unexplored. As illustrated in Figure 8, this study showed that BRAF is up-regulated in human heart failure and activation of BRAF in cardiomyocytes promotes cardiomyocyte hypertrophy. The data for BRAF in adult human heart failure shown in Figure 1 are intriguing, although (as with any studies of end-stage disease) it is difficult to know whether the changes are cause or consequence of the disease. Up-regulation of BRAF in separate cohorts of heart failure patients, with selective up-regulation of $B R A F$ and down-regulation of RAF1 and $A R A F$ isoforms in patients with dilated cardiomyopathy, suggests that the changes are a key feature of a failing heart. Even if BRAF is not a driving force in the development of human heart failure, an increase in BRAF expression (and potentially, BRAF activation) may influence the failing phenotype. It should be noted that RAF kinases are potentially expressed at different ratios and levels in different cell types, so the differences in expression of the RAF isoforms could simply result from changes in cellular content in failing hearts compared with the normal controls. In this case, the changes may sustain a failing phenotype and reduce potential for recovery. On the other hand, an increase in BRAF expression may reflect an increase in cardioprotective signalling. On balance, it seems appropriate to consider that the increase in BRAF expression in heart failure is potentially an important facet of the disease.

ERK1/2 were shown to be activated in cardiomyocytes by hypertrophic agonists as early as 1993 [45]. Subsequent work from many groups established that ERK1/2 signalling is important in cardiomyocyte hypertrophy, but also that it is cardioprotective [6-8]. Possibly the earliest publication on RAF kinases in the heart was in 1995 [10], but this focused on RAF1 and ARAF, the difficulty with BRAF being the lack of antibodies of sufficient quality to study BRAF properly in cardiac tissues. Although this was discussed, it became generally 
A

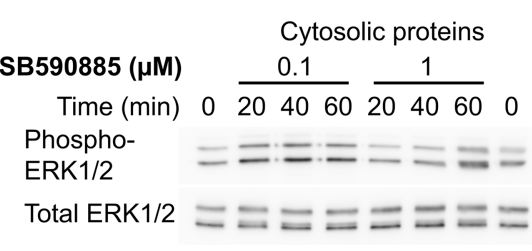

B

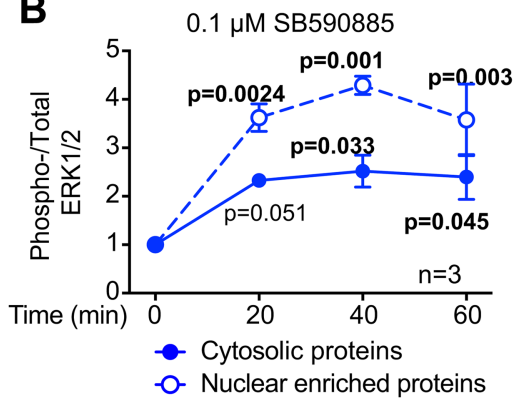

C

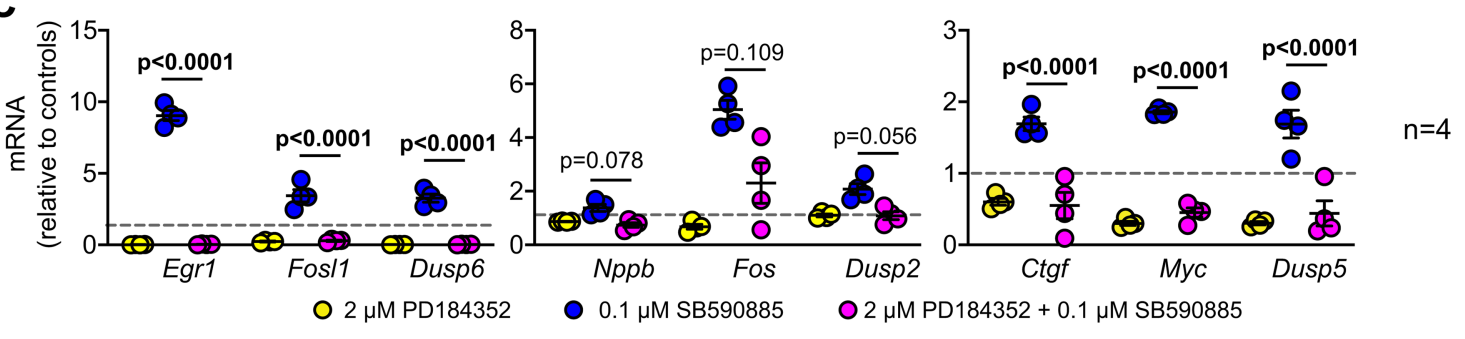

D
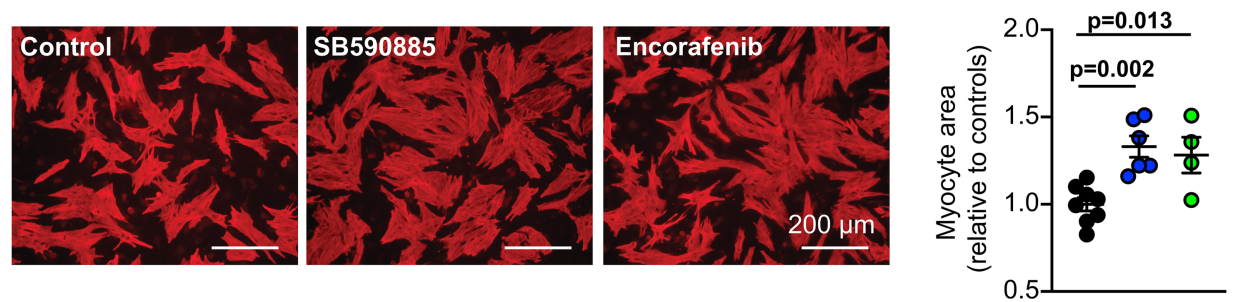

- Control

- SB590885

○ Encorafenib
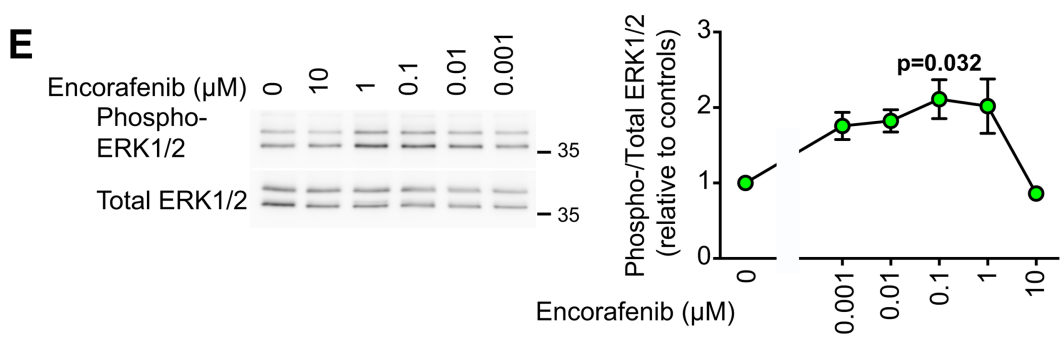

$\mathbf{F}$
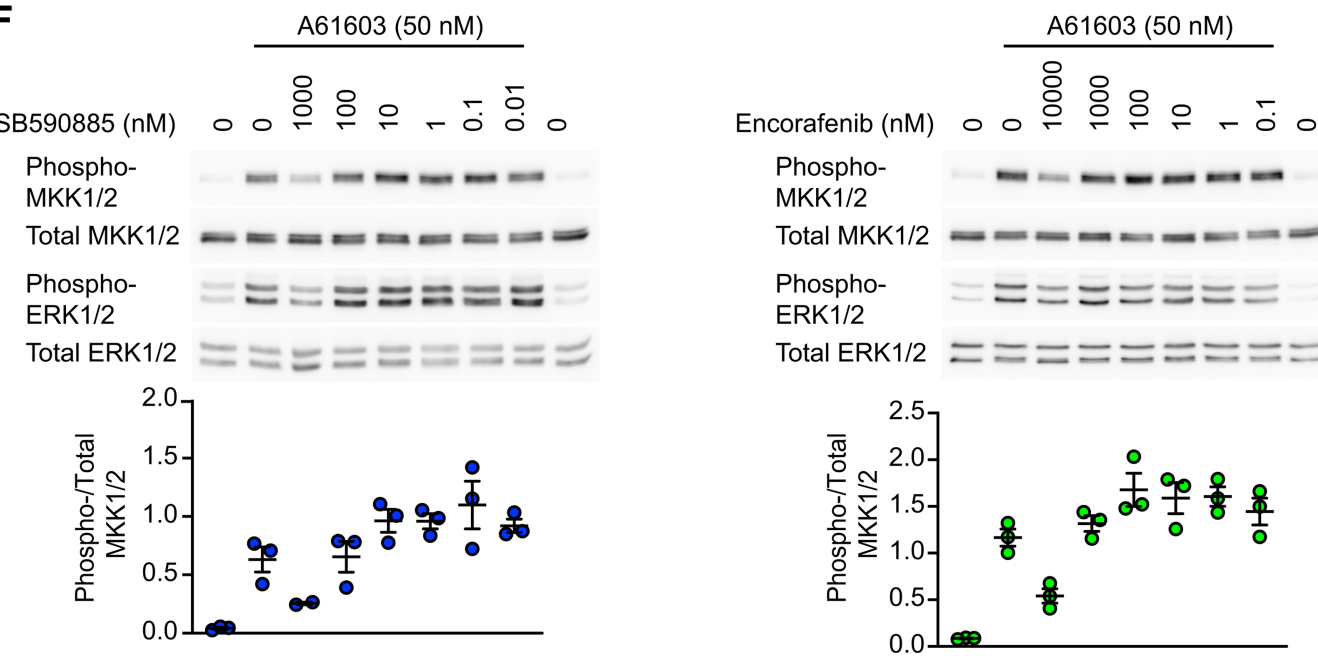

Figure 5. SB590885 promotes immediate early gene expression and hypertrophy in cardiomyocytes.

(A and B) Rat neonatal cardiomyocytes were exposed to the indicated concentrations of SB590885 for the times shown. Cytosolic and nuclear-enriched fractions were immunoblotted for phosphorylated (phospho-) or total ERK1/2. Representative blots are shown (A) with 

Holm-Sidak's post-test). Positions of relative molecular mass markers $(\mathrm{kDa})$ are on the right of the immunoblots. (C) Rat neonatal cardiomyocytes were untreated or exposed to $0.1 \mu \mathrm{M} \mathrm{SB590885}(1 \mathrm{~h})$ with/without $2 \mu \mathrm{M}$ PD184352. mRNA expression was measured by quantitative qPCR. Individual data points are shown with means $\pm \operatorname{SEM}(n=4)$. $P$ values are for PD184352/SB590885 vs SB590885 (one-way ANOVA with Holm-Sidak's post-test). (D) Cardiomyocytes were incubated with $1 \mu \mathrm{M}$ SB590885, $1 \mu \mathrm{M}$ encorafenib or under control conditions $(24 \mathrm{~h})$ and immunostained for troponin T. Images (left panels) are representative of 6 (SB590885, controls) or 4 (encorafenib) independent myocyte preparations with analysis of the surface area of individual cardiomyocytes shown in the right panel. Images are from the same experiment. Individual data points are shown for the mean values for cardiomyocyte area for each experiment, normalised to the mean value of the controls. We also show the means \pm SEM for all of the experiments. Statistical analysis used a two-tailed paired $t$-test. (E) Immunoblot analysis of phosphorylated (phospho-) and total ERK1/2 in extracts from neonatal rat cardiomyocytes exposed to the indicated concentrations of encorafenib $(20 \mathrm{~min})$. Representative blots are shown on the left with densitometric analysis on the right. Results are means \pm SEM ( $n=3$ independent cell preparations). $P$ value is relative to controls with no encorafenib (one-way ANOVA with Holm-Sidak's post-test). (F) Immunoblot analysis of phospho- and total MKK1/2 or ERK1/2 in extracts from neonatal rat cardiomyocytes exposed to the indicated concentrations of SB590885 or encorafenib (20 min) with addition of $50 \mathrm{nM} \mathrm{A61603}$ for the final $5 \mathrm{~min}$ as indicated. Representative blots are shown in the upper panels with densitometric analysis for MKK $1 / 2$ in the lower panels. Results are means $\pm \operatorname{SEM}(n=3$ independent cell preparations).

assumed that BRAF was not expressed at any significant levels in the heart. Assessment of the cardiomyocyte kinome showed quite clearly that BRAF is, indeed, expressed in cardiomyocytes [15]. Here, we show further that activation of cardiomyocyte BRAF alone is capable of driving hypertrophy, since activation of endogenous BRAF by knock-in of the V600E oncogenic mutation promotes cardiac and cardiomyocyte hypertrophy in vivo (Figures 2 and 3). Although there was increased mRNA expression of foetal gene markers ( $M y h 7, N p p a, N p p b$ ) and pro-fibrotic genes (IL11, Col1a1) generally associated with pathological hypertrophy, histological assessment of the myocardium showed little indication of any fibrosis (Figure 3). Indeed, the increase in left ventricular wall thickness and predicted mass appeared to be attributable solely to cardiomyocyte hypertrophy, with increased cardiomyocyte cross-sectional area and an associated enhancement of fractional shortening and ejection fraction.

The data with cardiomyocyte-specific $\mathrm{BRAF}^{\mathrm{V} 600 \mathrm{E}}$ knock-in are consistent with compensated, concentric hypertrophy in mice with cardiomyocyte-specific expression of constitutively-active MKK1 [46], and in accord with studies of mice with deletion of ERK1 and/or ERK2 in heart failure models showing that ERK1/2 signalling is cardioprotective and required for concentric hypertrophy [47]. Compensated 'physiological' hypertrophy is generally associated with activation of $\mathrm{mTORC} 1$ that increases protein synthesis acting, in part, through p70S6K [33,34]. However, we did not detect any increase in phosphorylation of Akt(T308) (the phosphorylation site associated with mTORC1 activation) or p70S6K in hearts from mice with cardiomyocyte-specific $\mathrm{BRAF}^{\mathrm{V} 600 \mathrm{E}}$ knock-in (Figure 2E). We did detect an increase in Akt(S473) phosphorylation, suggesting there was activation of mTORC2 that is predicted to affect gene expression. The mechanism for this is unclear, but ERK1/2 signalling can have an input into mTOR signalling and it is possible that there is a selective effect on mTORC2 rather than mTORC1. Irrespective of this, it appears that the ERK1/2 signal can promote physiological hypertrophy via an alternative route to mTORC1. Here, it should be considered that, although a dominant effect of the ERK1/2 cascade is to increase gene expression, it regulates the function of many proteins including the protein synthetic machinery $[48,49]$.

Cardiomyocyte knock-in of $\mathrm{BRAF}^{\mathrm{V} 600 \mathrm{E}}$ increased phosphorylation of $\mathrm{MKK} 1 / 2$ (immediately downstream of RAF kinases) and p90RSK (downstream of ERK1/2), and increased expression of ERK1/2-dependent genes (Figure 2). In this study, we assessed ERK1/2 activation using antibodies to a form which is dually phosphorylated on both the Thr and Tyr residues of the activation lip TEY motif, supposedly synonymous with activation. Confusingly, we have been unable to detect an increase in phosphorylation of ERK1/2 of this motif. A key factor for consideration is the effects of phosphatases. ERK1/2 phosphorylation of the TEY motif may be targeted by Ser/Thr, Tyr and dual-specificity phosphatases (DUSPs) that target both Thr/Tyr residues [50]. Although phosphorylation of both Thr and Tyr residues is required for full activity, there is residual activity of monophosphorylated forms (as we have previously shown [51]) and the likely mix of differentially 
A
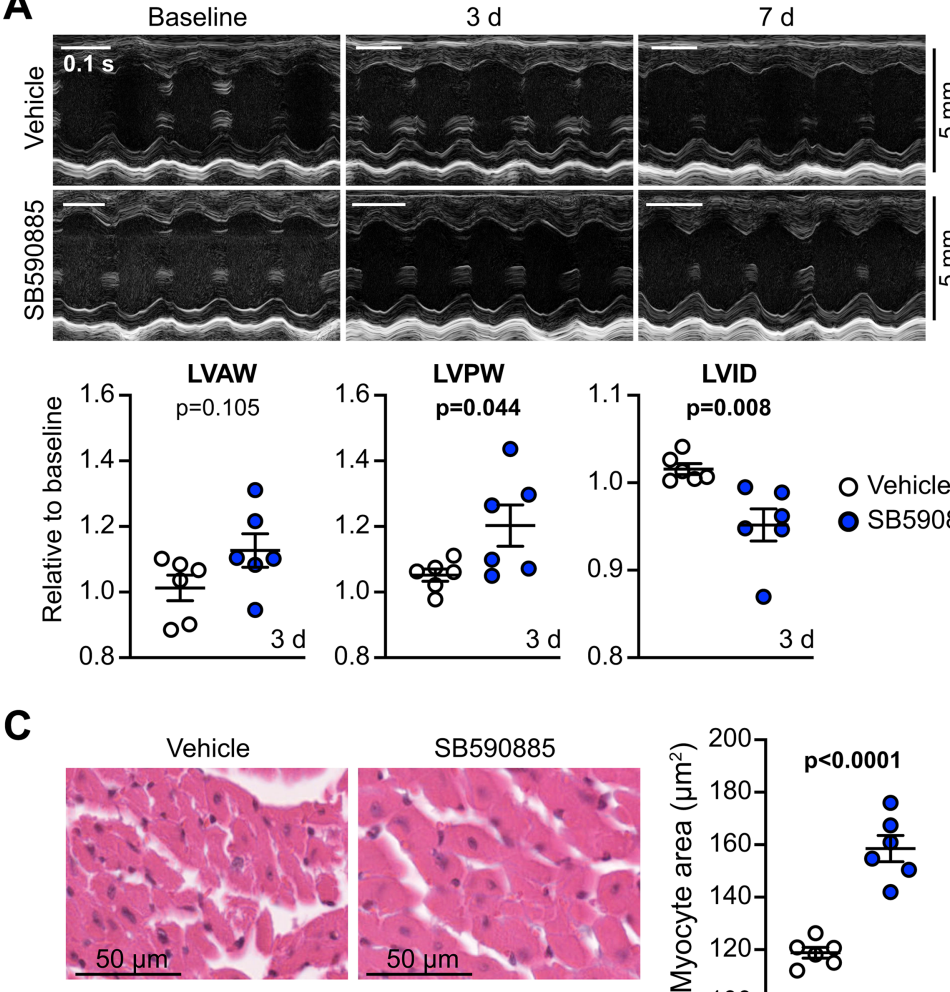

LVID

C
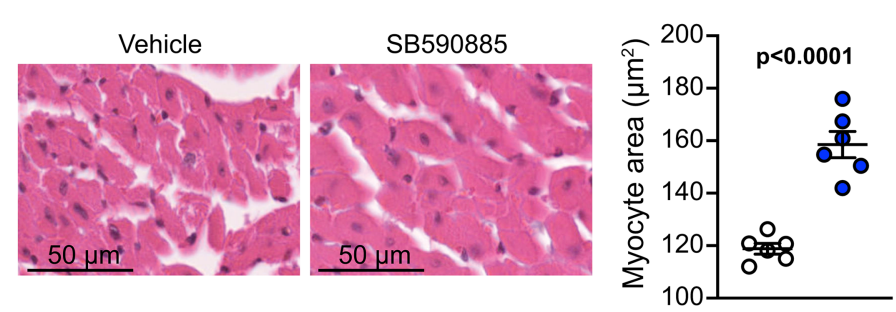

E

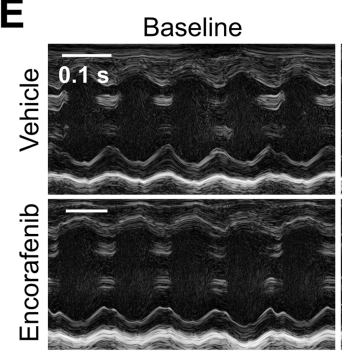

$3 d$
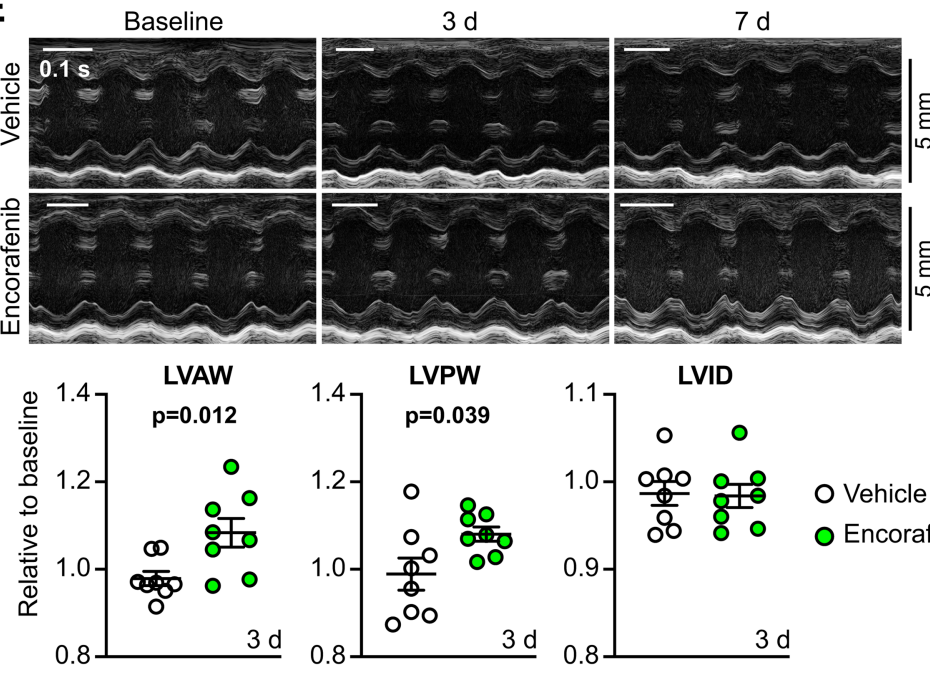

0.9
0.8 $\frac{0}{800}$

ㅇ O Vehicle
표 O SB590885
Encorafenib

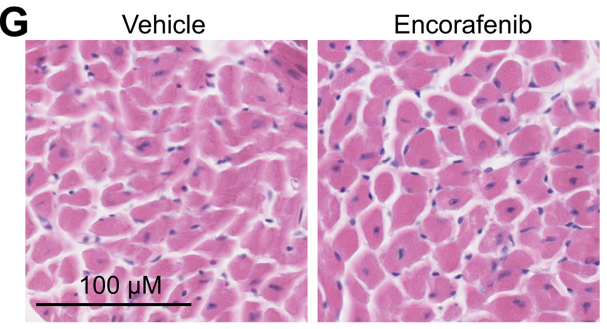

B
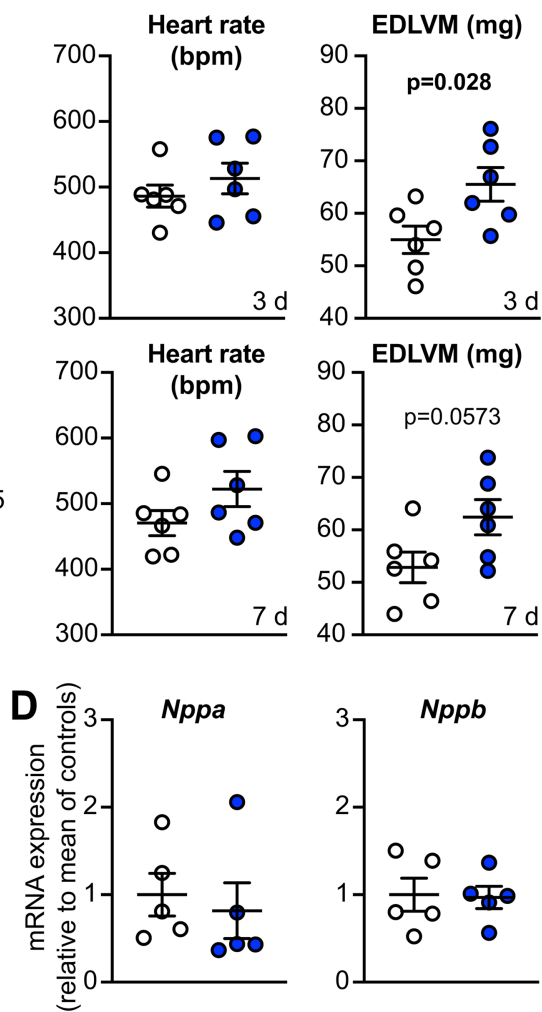

$\mathbf{F}$
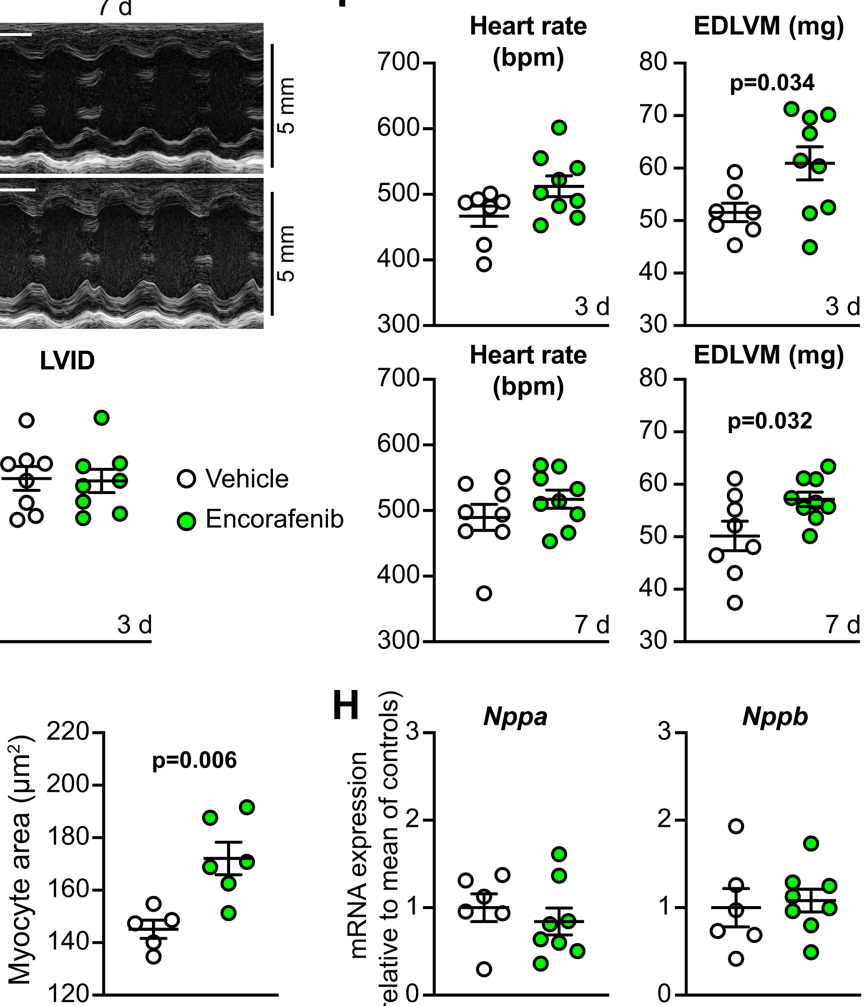

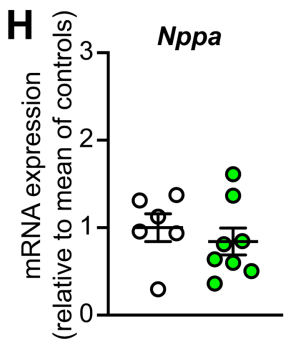

Figure 6. SB590885 and encorafenib promote cardiac hypertrophy in vivo.

Part 1 of 2

C57BI/6J mice were treated with vehicle, $0.5 \mathrm{mg} / \mathrm{kg} / \mathrm{d}$ SB590885 (A-D), or $3 \mathrm{mg} / \mathrm{kg} / \mathrm{d}$ encorafenib (E-H). Cardiac function and dimensions were measured by echocardiography. (A and $\mathbf{E}$ ) M-mode images from short-axis views of the heart were analysed to provide data for cardiac dimensions. 
Representative images are in the upper panels with analysis of echocardiograms shown below. LV, left ventricle; AW, anterior wall; PW, posterior wall; ID, internal diameter. Diastolic measurements are shown with all data provided in Supplementary Table S10. (B and F) B mode images from mice at $3 \mathrm{~d}$ (upper panels) or $7 \mathrm{~d}$ (lower panels) were subject to strain analysis using speckle-tracking to gain an estimate of left ventricular mass. EDLVM, end diastolic left ventricular mass. Additional data are provided in Supplementary Table S9. (C and G) Haemotoxylin (H\&E) and eosin staining of mouse heart sections.

Representative images are on the left with quantification of cardiomyocyte size from H\&E-stained sections on the right. Individual data points are shown with means \pm SEM. Statistical analysis used a two-tailed paired $t$-test. (D and H) RNA was isolated from hearts of mice treated with vehicle, SB590885 or encorafenib for $7 \mathrm{~d}$, and mRNA expression assessed by qPCR. Data are individual points with means \pm SEM.

phosphorylated species resulting from Ser/Thr or Tyr phosphatase activities makes it difficult to measure ERK1/2 activity using phospho-specific antibodies for immunoblotting. There is also likely to be changing expression of DUSPs, some of which are regulated downstream of ERK1/2 (e.g. DUSP 2, 6 and 5, as shown in Figure 5C). In non-synchronous cells in the in vivo model, these phosphatase activities may result in low-level 'flickering' of the ERK1/2 signal that is extremely difficult to detect even though the signal remains sufficient to drive downstream events. A second factor to consider is that we still do not know the full details of how ERK1/ 2 are regulated. There are other phosphorylation sites apart from the activation lip TEY motif that influence activity and may affect antibody recognition (e.g. flanking site inhibitory phosphorylations [52]), and there are other post-translational modifications. For example, a recent report indicates that ERK1/2 are dynamically regulated by S-palmitoylation downstream of MKK1/2, a modification which affects phosphorylation of the TEY motif but which is required for the full ERK1/2-dependent transcriptional programme [53]. Further studies are obviously required.

Activating the ERK1/2 cascade in cardiomyocytes may be beneficial in pathological situations that lead to heart failure. However, activating the pathway in fibroblasts is potentially less desirable since it may increase cardiac fibrosis [37,54]. Harnessing the benefits of the ERK1/2 pathway for the heart is therefore challenging: activation of protein kinase signalling is less straightforward than inhibition, and selective activation in one cell type over another is even more difficult. Our data suggest that Type 1 RAF inhibitors may be a way to do this. Thus, the established Type 1 inhibitor SB590885 activated ERK1/2 signalling in cultured cardiomyocytes and perfused hearts, increasing cardiomyocyte cross-sectional area in cells in culture and in hearts in vivo (Figures 4G-J, 5D and 6C). Similar effects were seen with encorafenib, a BRAF-targeted inhibitor used clinically, albeit generally in combination with a MKK1/2 inhibitor [38]. Our data suggest encorafenib is a Type 1 inhibitor, since it activated ERK1/2, in addition to increasing cardiomyocyte size both in cultured cells and in vivo (Figures 5D,E and 6G). The effects of SB590885 and encorafenib contrast with dabrafenib, a Type 1.5 inhibitor that, in our hands, has limited RAF paradox-inducing effects and does not induce cardiomyocyte hypertrophy in wild-type mice [21]. Indeed, dabrafenib inhibited fibrosis in the hearts of mice treated with angiotensin II to induce hypertension, suggesting that it serves as an inhibitor of signalling in the heart, rather than an activator. The mechanisms behind 'RAF paradox' signalling in cancer cells are reasonably well-investigated [20]: Type 1 inhibitors bind to RAF kinases in an active conformation, increasing dimerisation potential; Type 1.5 inhibitors bind a similar conformation, but with some alterations more consistent with an inactive enzyme. This could explain why SB590885/encorafenib and dabrafenib have different effects on the heart.

As terminally differentiated cells, cardiomyocytes may be viewed as having 'hard-wired' ERK1/2 signalling. This is reflected in the pre-formed RAF complexes detected in cardiomyocytes even from neonatal hearts (Figure 4A-E), suggesting that cardiomyocytes are primed for RAF signalling, enabling them to respond rapidly to extracellular stimuli. Consistent with this, the ERK1/2 cascade can be activated within 3 min of stimulation with receptor agonists (e.g. epidermal growth factor or endothelin-1 [11]), with up-regulation of the first ERK1/2-dependent immediate early genes within 15-30 min [35]. This rapid signalling may be facilitated by pre-formed signalling complexes. Such 'signalosomes' are established for the ERK1/2 cascade itself [55], and the presence of ERK1/2 signalosomes in cardiomyocytes could account for rapid activation in association with receptor stimulation, but also allow for the relatively slow effect of SB590885 which induces BRAF paradox signalling over 10-20 min (Figure 4H,I). For the latter, the drug needs to diffuse into cells and permeate intracellular compartments before becoming effective. SB590885 not only activated ERK1/2 in the 


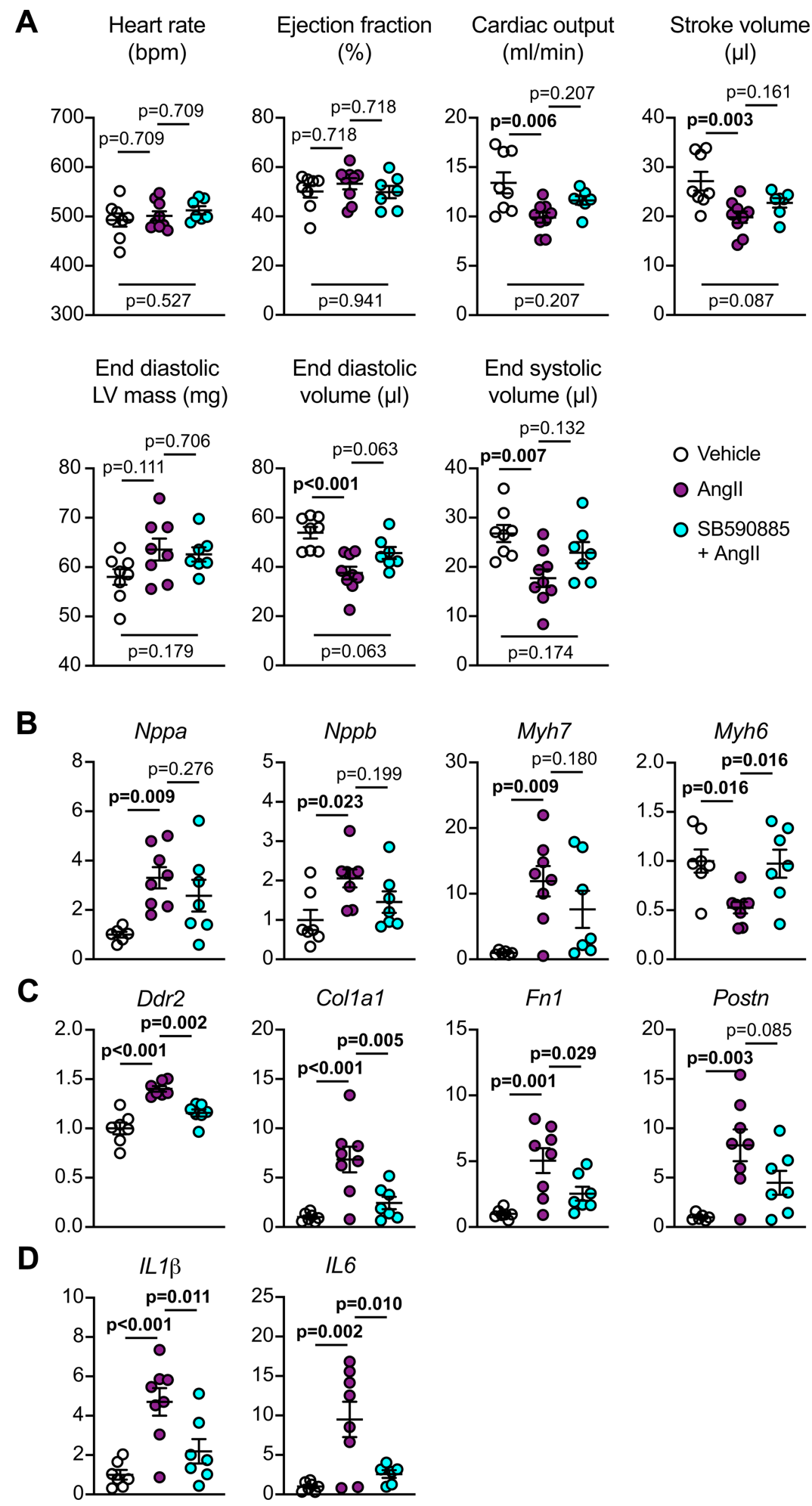

Figure 7. Effects of SB590885 on hypertension-induced cardiac hypertrophy in mice in vivo.

C57BI/6J mice were treated with vehicle, $0.8 \mathrm{mg} / \mathrm{kg} / \mathrm{d}$ angiotensin II (Angll) or Angll with $0.5 \mathrm{mg} / \mathrm{kg} / \mathrm{d}$ SB590885 for $7 \mathrm{~d}$. (A) Cardiac function and dimensions were measured by echocardiography with speckle-tracking analysis of B-mode long axis images. (B-D) RNA was isolated from hearts of mice treated with vehicle, Angll or Angll with SB590885, and mRNA expression assessed by qPCR. Data are individual points with means \pm SEM. Statistical analysis: one-way ANOVA with Holm-Sidak's post-test. 


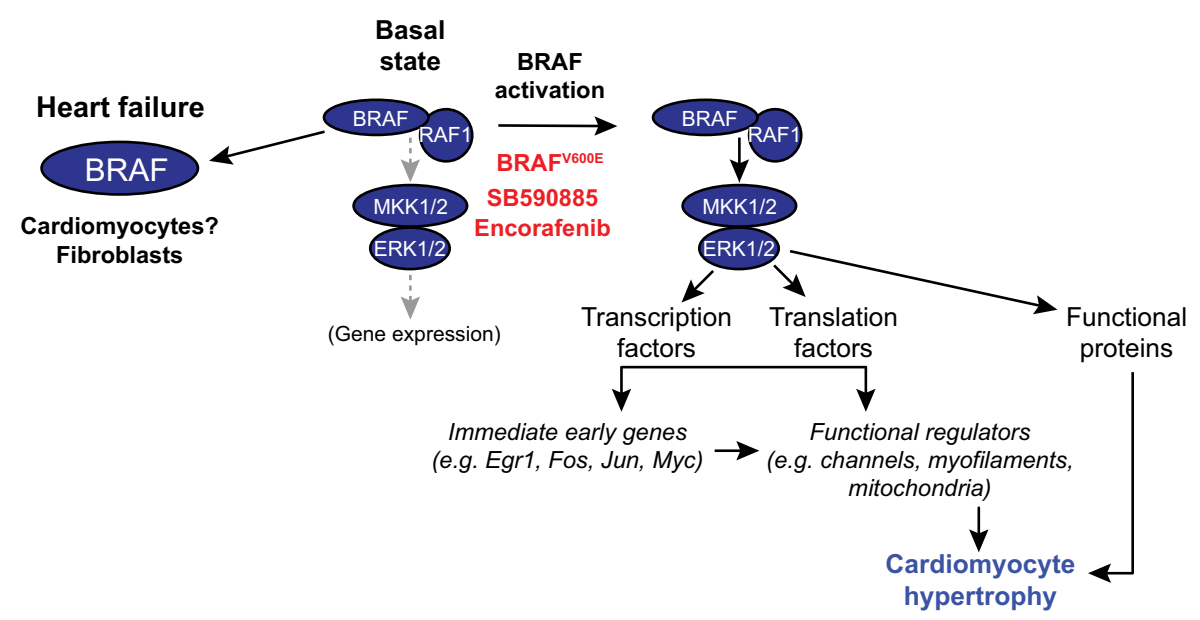

Figure 8. Schematic representation of the conclusions from this study.

BRAF is up-regulated in human failing hearts, although the cells involved remain to be defined. In cardiomyocytes, BRAF and RAF1 exist in preformed complexes. Activation of cardiomyocyte BRAF by knock-in of the V600E mutation or with Type 1 RAF kinase 'inhibitors' (e.g. SB590885, encorafenib) results in activation of MKK1/2 (mitogen-activated protein kinase kinases 1 and 2) and ERK1/2 (extracellular signal-regulated kinases 1 and 2). This leads to phosphorylation and activation of a range of substrates to modify activities of functional proteins in the cell, along with transcription and translation factors that promote expression of immediate early genes with stimulation of downstream gene expression, resulting in cardiomyocyte hypertrophy.

cytoplasm, but also increased activated ERK1/2 in the nucleus (Figure 5A,B). As in our previous studies of endothelin-1 [36], we did not detect net translocation of ERK1/2 to the nucleus following stimulation with SB590885. Interestingly, we had greater relative activation in the nucleus than the cytoplasm compared with endothelin-1, potentially resulting from free diffusion of the drug rather than receptor-driven signalling. This raises the intriguing possibility that the ERK1/2 cascade in cardiomyocytes is hard-wired to such a degree that ERK1/2 signalling is strictly compartmentalised, and signalling in the nucleus is divorced from that in the cytoplasm. Consistent with the presence of nuclear-activated ERK1/2, SB590885 promoted ERK1/2-dependent changes in immediate early gene expression and induced an increase in cardiomyocyte size (Figure 5C,D). This is in accord with the body of evidence implicating ERK1/2 signalling in cardiomyocyte hypertrophy [56].

Anti-cancer therapies are designed to prevent cell growth/proliferation and promote death of cancerous cells, and this has driven much of the pioneering research into intracellular signalling and regulation of cell function. The requirements for cancer therapies are generally diametrically opposed to the requirements of an adult heart subjected to pathophysiological stresses, in which cytoprotection and increased cardiomyocyte growth (if not proliferation) are desirable [57]. It is therefore unsurprising that cancer therapies have cardiotoxic effects both during cancer treatment and in cancer survivors [58,59]. MKK1/2 inhibitors clearly have cardiotoxic effects and trametinib causes hypertension in $\sim 26 \%$ of patients with decreased ejection fraction in $7-11 \%$ of patients $[60,61]$. This is likely to be an on-target effect since alternative MKK1/2 inhibitors (selumetinib and cobimetinib) are also associated with increased risk of hypertension and reduced ejection fraction [62]. The situation with RAF inhibitors is less clear. Vemurafenib increases QT interval, but dabrafenib monotherapy has not been associated with any obvious cardioxicity [63-65], suggesting that the effects of vemurafenib are unrelated to inhibition of RAF kinases. Unlike MKK1/2 inhibitors, Raf inhibitors alone are not reported to cause hypertension. [65]. Indeed, our studies suggest that they do not affect blood pressure since SB590885 and encorafenib promoted an increase in cardiomyocyte size (indicating that there was no unloading of the heart resulting from lowered blood pressure) in the absence of any fibrosis or changes in gene expression associated with hypertension (Figures 6 and 7; [21]). Encorafenib has not yet been used extensively as a monotherapy, being used clinically in combination with the MKK1/2 inhibitor binimetinib [43], but our data suggest that, by activating the 'RAF paradox' in cardiomyocytes, encorafenib is more likely to be beneficial for the heart, rather than detrimental. With any small molecule inhibitor, there is always consideration of off-target effects. However, we used two different compounds to induce the 'RAF paradox' (SB590885 and encorafenib), both of 
which promoted cardiomyocyte hypertrophy in vitro and in vivo (Figures 4-6), suggesting the response is an on-target effect.

Overall, this study provides data to demonstrate that selective activation of ERK1/2 signalling in cardiomyocytes (potentially desirable in a failing heart) can be achieved. The Type 1 RAF inhibitors being used for cancer are not cardiotoxic per se and, with further research, they may even prove useful as a supporting therapy in failing hearts. Our data with SB590885 in a mouse model of hypertension suggest this could, indeed, be the case, since SB590885 appeared to improve left ventricular function to some degree and it suppressed the increase in expression of pro-fibrotic and pro-inflammatory genes (Figure 7). However, further work is necessary. Since SB590885 is a drug that has not progressed in human clinical trials, we have terminated these experiments on the basis that it would be preferable to study encorafenib as a drug that is already in clinical use. Assuming RAF inhibitors that activated ERK1/2 in cardiomyocytes are useful for heart failure, it is necessary to consider whether using drugs to enhance ERK1/2 signalling for heart failure could increase the potential for unregulated cell proliferation or even cancer. One side effect of RAF inhibitors is the development of squamous cell carcinoma [16], but this is manageable in the context of cancer therapy. It may also be appropriate to limit the duration of treatment to restrict the possibility of cancers developing. However, heart failure is just as devastating a disease as cancer, and the benefits afforded potentially outweigh the risks in this setting.

\section{Data Availability}

All primary data are available from the corresponding author upon reasonable request. Additional data sharing information is not applicable to this study.

\section{Competing Interests}

The authors declare that there are no competing interests associated with the manuscript.

\section{Funding}

This work was funded by the British Heart Foundation (Grant Nos.: PG/13/71/30460, PG/17/11/32841, PG/15/ 24/31367, PG/15/31/31393, FS/18/33/33621, FS/19/24/34262, PG/19/7/34167, PG/19/32/34383), the Wellcome Trust (Grant No.: 204809/Z/16/Z), Fondation Leducq (Grant No.: CV05-02), NIHR Imperial College Biomedical Research Centre, and Qassim University, Saudi Arabia (H.O.A.).

\section{Open Access Statement}

Open access for this article was enabled by the participation of University of Reading in an all-inclusive Read \& Publish pilot with Portland Press and the Biochemical Society under a transformative agreement with JISC.

\section{CRediT Author Contribution}

Angela Clerk: Conceptualization, Resources, Data curation, Formal analysis, Supervision, Funding acquisition, Investigation, Visualization, Writing - original draft, Project administration. Daniel N. Meijles: Resources, Data curation, Formal analysis, Investigation. Michelle A. Hardyman: Formal analysis, Investigation, Methodology. Stephen J. Fuller: Investigation, Methodology. Sonia P. Chothani: Investigation. Joshua J. Cull: Investigation. Susanna T.E. Cooper: Investigation. Hajed O. Alharbi: Investigation. Konstantinos Vanezis: Investigation. Leanne E. Felkin: Investigation. Thomais Markou: Investigation. Samuel J. Leonard: Methodology. Spencer W. Shaw: Investigation. Owen J.L. Rackham: Supervision, Investigation. Stuart A. Cook: Resources, Supervision. Peter E. Glennon: Supervision, Methodology. Mary N. Sheppard: Resources, Supervision. John C. Sembrat: Resources. Mauricio Rojas: Resources. Charles F. McTiernan: Resources. Paul J. Barton: Resources, Data curation, Supervision. Peter H. Sugden: Conceptualization, Resources, Supervision, Funding acquisition, Writing - original draft.

\section{Abbreviations}

ERK1/2, extracellular signal-regulated kinase; LV, left ventricular; MAPK, mitogen-activated protein kinase; MKK, MAPK kinase.

\section{References}

1 Ahuja, P., Sdek, P. and MacLellan, W.R. (2007) Cardiac myocyte cell cycle control in development, disease, and regeneration. Physiol. Rev. 87, 521-544 https://doi.org/10.1152/physrev.00032.2006 
2 Dorn, II, G.W., Robbins, J. and Sugden, P.H. (2003) Phenotyping hypertrophy: eschew obfuscation. Circ. Res. 92, 1171-1175 https://doi.org/10.1161/ 01.RES.0000077012.11088.BC

3 Zhang, J., Liu, D., Zhang, M. and Zhang, Y. (2019) Programmed necrosis in cardiomyocytes: mitochondria, death receptors and beyond. Br. J. Pharmacol. 176, 4319-4339 https://doi.org/10.1111/bph.14363

4 Teo, L.Y.L., Chan, L.L. and Lam, C.S.P. (2016) Heart failure with preserved ejection fraction in hypertension. Curr. Opin. Cardiol. 31, 410-416 https://doi.org/10.1097/HC0.0000000000000292

5 Suthahar, N., Meijers, W.C., Sillje, H.H.W. and de Boer, R.A. (2017) From inflammation to fibrosis-molecular and cellular mechanisms of myocardia tissue remodelling and perspectives on differential treatment opportunities. Curr. Heart Fail. Rep. 14, 235-250 https://doi.org/10.1007/ s11897-017-0343-y

6 Sugden, P.H. and Clerk, A. (1998) Cellular mechanisms of cardiac hypertrophy. J. Mol. Med. 76, 725-746 https://doi.org/10.1007/s001090050275

7 Rose, B.A., Force, T. and Wang, Y. (2010) Mitogen-activated protein kinase signaling in the heart: angels versus demons in a heart-breaking tale Physiol. Rev. 90, 1507-1546 https://doi.org/10.1152/physrev.00054.2009

8 Kehat, I. and Molkentin, J.D. (2010) Extracellular signal-regulated kinase 1/2 (ERK1/2) signaling in cardiac hypertrophy. Ann. N. Y. Acad. Sci. 1188, 96-102 https://doi.org/10.1111/j.1749-6632.2009.05088.x

9 Matallanas, D., Birtwistle, M., Romano, D., Zebisch, A., Rauch, J., von Kriegsheim, A. et al. (2011) Raf family kinases: old dogs have learned new tricks. Genes Cancer 2, 232-260 https://doi.org/10.1177/1947601911407323

10 Bogoyevitch, M.A., Marshall, C.J. and Sugden, P.H. (1995) Hypertrophic agonists stimulate the activities of the protein kinases c-Raf and A-Raf in cultured ventricular myocytes. J. Biol. Chem. 270, 26303-26310 https://doi.org/10.1074/jbc.270.44.26303

11 Clerk, A., Aggeli, I.K.S., Stathopoulou, K. and Sugden, P.H. (2006) Peptide growth factors signal differentially through protein kinase C to extracellular signal-regulated kinases in neonatal cardiomyocytes. Cell. Signal. 18, 225-235 https://doi.org/10.1016/j.cellsig.2005.04.005

12 Chiloeches, A., Paterson, H.F., Marais, R., Clerk, A., Marshall, C.J. and Sugden, P.H. (1999) Regulation of Ras.GTP loading and Ras-Raf association in neonatal rat ventricular myocytes by $G$ protein-coupled receptor agonists and phorbol ester. activation of the ERK cascade by phorbol ester is mediated by Ras. J. Biol. Chem. 274, 19762-19770 https://doi.org/10.1074/jbc.274.28.19762

13 Yamaguchi, O., Watanabe, T., Nishida, K., Kishiwase, K., Higuchi, Y., Takeda, T. et al. (2004) Cardiac-specific disruption of the c-raf-1 gene induces cardiac dysfunction and apoptosis. J. Clin. Invest. 114, 937-943 https://doi.org/10.1172/JCl200420317

14 Harris, I.S., Zhang, S., Treskov, I., Kovacs, A., Weinheimer, C. and Muslin, A.J. (2004) Raf-1 kinase is required for cardiac hypertrophy and cardiomyocyte survival in response to pressure overload. Circulation 110, 718-723 https://doi.org/10.1161/01.CIR.0000138190. 50127.6A

15 Fuller, S.J., Osborne, S.A., Leonard, S.J., Hardyman, M.A., Vaniotis, G., Allen, B.G. et al. (2015) Cardiac protein kinases: the cardiomyocyte kinome and differential kinase expression in human failing hearts. Cardiovasc. Res. 108, 87-98 https://doi.org/10.1093/cvr/cw210

16 Roskoski, Jr, R. (2018) Targeting oncogenic Raf protein-serine/threonine kinases in human cancers. Pharmacol. Res. 135, 239-258 https://doi.org/10 1016/.jphrs.2018.08.013

17 Dhomen, N. and Marais, R. (2007) New insight into BRAF mutations in cancer. Curr. Opin. Genet. Dev. 17, 31-39 https://doi.org/10.1016/j.gde.2006. 12.005

18 Roring, M. and Brummer, T. (2012) Aberrant B-Raf signaling in human cancer - 10 years from bench to bedside. Crit. Rev. Oncog. 17, 97-121 https://doi.org/10.1615/CritRevOncog.v17.11.70

19 Rheault, T.R., Stellwagen, J.C., Adjabeng, G.M., Hornberger, K.R., Petrov, K.G., Waterson, A.G. et al. (2013) Discovery of dabrafenib: a selective inhibitor of Raf kinases with antitumor activity against B-Raf-driven tumors. ACS Med. Chem. Lett. 4, 358-362 https://doi.org/10.1021/ $\mathrm{m} \mid 4000063$

20 Durrant, D.E. and Morrison, D.K. (2018) Targeting the Raf kinases in human cancer: the Raf dimer dilemma. Br. J. Cancer 118, 3-8 https://doi.org/10. 1038/bjc.2017.399

21 Meijles, D.N., Cull, J.J., Cooper, S.T.E., Markou, T., Hardyman, M.A., Fuller, S.J. et al. (2021) The anti-cancer drug dabrafenib is not cardiotoxic and inhibits cardiac remodelling and fibrosis in a murine model of hypertension. Clin. Sci. (Lond) 135, 1631-1647 https://doi.org/10.1042/CS20210192

22 Heinig, M., Adriaens, M.E., Schafer, S., van Deutekom, H.W.M., Lodder, E.M., Ware, J.S. et al. (2017) Natural genetic variation of the cardiac transcriptome in non-diseased donors and patients with dilated cardiomyopathy. Genome Biol. 18, 170 https://doi.org/10.1186/s13059-017-1286-z

23 Love, M.I., Huber, W. and Anders, S. (2014) Moderated estimation of fold change and dispersion for RNA-seq data with DESeq2. Genome Biol. 15, 550 https://doi.org/10.1186/s13059-014-0550-8

24 Dankort, D., Filenova, E., Collado, M., Serrano, M., Jones, K. and McMahon, M. (2007) A new mouse model to explore the initiation, progression, and therapy of BRAFV600E-induced lung tumors. Genes Dev. 21, 379-384 https://doi.org/10.1101/gad.1516407

25 Sohal, D.S., Nghiem, M., Crackower, M.A., Witt, S.A., Kimball, T.R., Tymitz, K.M. et al. (2001) Temporally regulated and tissue-specific gene manipulations in the adult and embryonic heart using a tamoxifen-inducible Cre protein. Circ. Res. 89, 20-25 https://doi.org/10.1161/hh1301.092687

26 Meijles, D.N., Cull, J.J., Markou, T., Cooper, S.T.E., Haines, Z.H.R., Fuller, S.J. et al. (2020) Redox regulation of cardiac ASK1 (Apoptosis Signal-regulating Kinase 1) controls p38-MAPK (mitogen-activated protein kinase) and orchestrates cardiac remodeling to hypertension. Hypertension 76 1208-1218 https://doi.org/10.1161/HYPERTENSIONAHA.119.14556

27 Meijles, D., Fuller, S.J., Cull, J.J., Alharbi, H.O., Cooper, S.T.E., Sugden, P.H. et al. (2021) The insulin receptor family and protein kinase B (Akt) are activated in the heart by alkaline $\mathrm{pH}$ and $\left(\alpha_{1}\right.$-adrenergic receptors. Biochem. J. 478, 2059-2079 https://doi.org/10.1042/BCJ20210144

28 Marshall, A.K., Barrett, O.P.T., Cullingford, T.E., Shanmugasundram, A., Sugden, P.H. and Clerk, A. (2010) ERK1/2 signaling dominates over rhoA signaling in regulating early changes in RNA expression induced by endothelin-1 in neonatal rat cardiomyocytes. PLOS ONE 5, e10027 https://doi.org/ 10.1371/journal.pone.0010027

29 Clerk, A., Pham, F.H., Fuller, S.J., Sahai, E., Aktories, K., Marais, R. et al. (2001) Regulation of mitogen-activated protein kinases in cardiac myocytes through the small G protein, Rac1. Mol. Cell. Biol. 21, 1173-1184 https://doi.org/10.1128/MCB.21.4.1173-1184.2001

30 Hougen, K., Aronsen, J.M., Stokke, M.K., Enger, U., Nygard, S., Andersson, K.B. et al. (2010) Cre-loxP DNA recombination is possible with only minimal unspecific transcriptional changes and without cardiomyopathy in Tg(alphaMHC-MerCreMer) mice. Am. J. Physiol. Heart Circ. Physiol. 299 H1671-H1678 https://doi.org/10.1152/ajpheart.01155.2009 
31 Jahn, H.M., Kasakow, C.V., Helfer, A., Michely, J., Verkhratsky, A., Maurer, H.H. et al. (2018) Refined protocols of tamoxifen injection for inducible DNA recombination in mouse astroglia. Sci. Rep. 8, 5913 https://doi.org/10.1038/s41598-018-24085-9

32 Romeo, Y., Zhang, X. and Roux, P.P. (2012) Regulation and function of the RSK family of protein kinases. Biochem. J. 441, 553-569 https://doi.org/ 10.1042/BJ20110289

33 Fenton, T.R. and Gout, I.T. (2011) Functions and regulation of the $70 \mathrm{kDa}$ ribosomal S6 kinases. Int. J. Biochem. Cell Biol. 43, 47-59 https://doi.org/ 10.1016/j.biocel.2010.09.018

34 Hay, N. (2011) Interplay between FOXO, TOR, and Akt. Biochim. Biophys. Acta 1813, 1965-1970 https://doi.org/10.1016/j.bbamcr.2011.03.013

35 Cullingford, T.E., Markou, T., Fuller, S.J., Giraldo, A., Pikkarainen, S., Zoumpoulidou, G. et al. (2008) Temporal regulation of expression of immediate early and second phase transcripts by endothelin-1 in cardiomyocytes. Genome Biol. 9, R32 https://doi.org/10.1186/ gb-2008-9-2-r32

36 Amirak, E., Fuller, S.J., Sugden, P.H. and Clerk, A. (2013) P90 ribosomal S6 kinases play a significant role in early gene regulation in the cardiomyocyte response to Gq protein-coupled receptor stimuli, endothelin-1 and $\left(\alpha_{1}\right.$-adrenergic receptor agonists. Biochem. J. 450, 351-363 https://doi.org/10. 1042/BJ20121371

37 Schafer, S., Viswanathan, S., Widjaja, A.A., Lim, W.W., Moreno-Moral, A., DeLaughter, D.M. et al. (2017) IL-11 is a crucial determinant of cardiovascular fibrosis. Nature 552, 110-115 https://doi.org/10.1038/nature24676

38 Rebocho, A.P. and Marais, R. (2012) ARAF acts as a scaffold to stabilize BRAF:CRAF heterodimers. Oncogene 32, 3207-3212 https://doi.org/10.1038/ onc. 2012.330

39 Li, F., Wang, X., Capasso, J.M. and Gerdes, A.M. (1996) Rapid transition of cardiomyocytes from hyperplasia to hypertrophy during postnatal development. J. Mol. Cell. Cardiol. 28, 1737-1746 https://doi.org/10.1006/jmcc.1996.0163

40 Yahalom-Ronen, Y., Rajchman, D., Sarig, R., Geiger, B. and Tzahor, E. (2015) Reduced matrix rigidity promotes neonatal cardiomyocyte dedifferentiation, proliferation and clonal expansion. eLife 4, e07455 https://doi.org/10.7554/eLife.07455

41 King, A.J., Patrick, D.R., Batorsky, R.S., Ho, M.L., Do, H.T., Zhang, S.Y. et al. (2006) Demonstration of a genetic therapeutic index for tumors expressing oncogenic BRAF by the kinase inhibitor SB-590885. Cancer Res. 66, 11100-11105 https://doi.org/10.1158/0008-5472.CAN-06-2554

42 Davies, S.P., Reddy, H., Caivano, M. and Cohen, P. (2000) Specificity and mechanism of action of some commonly used protein kinase inhibitors. Biochem. J. 351, 95-105 https://doi.org/10.1042/bj3510095

43 Roviello, G., D'Angelo, A., Petrioli, R., Roviello, F., Cianchi, F., Nobili, S. et al. (2020) Encorafenib, binimetinib, and cetuximab in BRAF V600E-mutated colorectal cancer. Transl. Oncol. 13, 100795 https://doi.org/10.1016/j.tranon.2020.100795

44 Valero-Munoz, M., Backman, W. and Sam, F. (2017) Murine models of heart failure with preserved ejection fraction: a 'fishing expedition'. JACC Basic Transl. Sci. 2, 770-789 https://doi.org/10.1016/j.jacbts.2017.07.013

45 Bogoyevitch, M.A., Glennon, P.E. and Sugden, P.H. (1993) Endothelin-1, phorbol esters and phenylephrine stimulate MAP kinase activities in ventricular cardiomyocytes. FEBS Lett. 317, 271-275 https://doi.org/10.1016/0014-5793(93)81291-7

46 Bueno, O.F., De Windt, L.J., Tymitz, K.M., Witt, S.A., Kimball, T.R., Klevitsky, R. et al. (2000) The MEK1-ERK1/2 signaling pathway promotes compensated cardiac hypertrophy in transgenic mice. EMBO J. 19, 6341-6350 https://doi.org/10.1093/emboj/19.23.6341

47 Kehat, I., Davis, J., Tiburcy, M., Accornero, F., Saba-El-Leil, M.K., Maillet, M. et al. (2011) Extracellular signal-regulated kinases 1 and 2 regulate the balance between eccentric and concentric cardiac growth. Circ. Res. 108, 176-183 https://doi.org/10.1161/CIRCRESAHA.110.231514

48 Yoon, S. and Seger, R. (2006) The extracellular signal-regulated kinase: multiple substrates regulate diverse cellular functions. Growth Factors 24, 21-44 https://doi.org/10.1080/02699050500284218

49 Proud, C.G. (2004) Ras, PI3-kinase and mTOR signaling in cardiac hypertrophy. Cardiovasc. Res. 63, 403-413 https://doi.org/10.1016/j.cardiores. 2004.02.003

50 Kidger, A.M. and Keyse, S.M. (2016) The regulation of oncogenic Ras/ERK signalling by dual-specificity mitogen activated protein kinase phosphatases (MKPs). Semin. Cell Dev. Biol. 50, 125-132 https://doi.org/10.1016/j.semcdb.2016.01.009

51 Sugden, P.H., Markou, T., Fuller, S.J., Tham, E.L., Molkentin, J.D., Paterson, H.F. et al. (2011) Monophosphothreonyl extracellular signal-regulated kinases 1 and 2 (ERK1/2) are formed endogenously in intact cardiac myocytes and are enzymically active. Cell Signal. 23, 468-477 https://doi.org/10 1016/j.cellsig.2010.10.024

52 Lai, S. and Pelech, S. (2016) Regulatory roles of conserved phosphorylation sites in the activation T-loop of the MAP kinase ERK1. Mol. Biol. Cell 27, 1040-1050 https://doi.org/10.1091/mbc.E15-07-0527

53 Azizi, S.A., Qui, T., Brookes, N. and Dickinson, B.C. (2021) Regulation of ERK2 activity by dynamic S-acylation. BioRXiv https://doi.org/10.1101/2021. 11.05.467491

54 Thum, T., Gross, C., Fiedler, J., Fischer, T., Kissler, S., Bussen, M. et al. (2008) MicroRNA-21 contributes to myocardial disease by stimulating MAP kinase signalling in fibroblasts. Nature 456, 980-984 https://doi.org/10.1038/nature07511

55 Kyriakis, J.M. (2007) The integration of signaling by multiprotein complexes containing Raf kinases. Biochim. Biophys. Acta 1773, 1238-1247 https://doi.org/10.1016/j.bbamcr.2006.11.003

56 Clerk, A., Cullingford, T.E., Fuller, S.J., Giraldo, A., Markou, T., Pikkarainen, S. et al. (2007) Signaling pathways mediating cardiac myocyte gene expression in physiological and stress responses. J. Cell Physiol. 212, 311-322 https://doi.org/10.1002/jcp.21094

57 Tarone, G., Balligand, J.L., Bauersachs, J., Clerk, A., de Windt, L., Heymans, S. et al. (2014) Targeting myocardial remodelling to develop novel therapies for heart failure: a position paper from the Working Group on Myocardial Function of the European Society of Cardiology. Eur. J. Heart Fail. 16, 494-508 https://doi.org/10.1002/ejhf.62

58 Force, T. and Kolaja, K.L. (2011) Cardiotoxicity of kinase inhibitors: the prediction and translation of preclinical models to clinical outcomes. Nat. Rev Drug Discov. 10, 111-126 https://doi.org/10.1038/nrd3252

59 Finet, J.E. (2017) Management of heart failure in cancer patients and cancer survivors. Heart Fail. Clin. 13, 253-288 https://doi.org/10.1016/j.hfc. 2016.12.004

60 Banks, M., Crowell, K., Proctor, A. and Jensen, B.C. (2017) Cardiovascular effects of the MEK inhibitor, trametinib: a case report, literature review, and consideration of mechanism. Cardiovasc. Toxicol. 17, 487-493 https://doi.org/10.1007/s12012-017-9425-z 
61 Bronte, E., Bronte, G., Novo, G., Rinaldi, G., Bronte, F., Passiglia, F. et al. (2018) Cardiotoxicity mechanisms of the combination of BRAF-inhibitors and MEK-inhibitors. Pharmacol. Ther. 192, 65-73 https://doi.org/10.1016/j.pharmthera.2018.06.017

62 Abdel-Rahman, O., ElHalawani, H. and Ahmed, H. (2015) Risk of selected cardiovascular toxicities in patients with cancer treated with MEK inhibitors: a comparative systematic review and meta-analysis. J. Glob. Oncol. 1, 73-82 https://doi.org/10.1200/JG0.2015.000802

63 Jordan, E.J. and Kelly, C.M. (2012) Vemurafenib for the treatment of melanoma. Expert Opin. Pharmacother. 13, 2533-2543 https://doi.org/10.1517/ 14656566.2012 .737780

64 Mincu, R.I., Mahabadi, A.A., Michel, L., Mrotzek, S.M., Schadendorf, D., Rassaf, T. et al. (2019) Cardiovascular adverse events associated with BRAF and MEK inhibitors: a systematic review and meta-analysis. JAMA Netw. Open 2, e198890 https://doi.org/10.1001/jamanetworkopen.2019.8890

65 Guha, A., Jain, P., Fradley, M.G., Lenihan, D., Gutierrez, J.M., Jain, C. et al. (2021) Cardiovascular adverse events associated with BRAF versus BRAF/ MEK inhibitor: cross-sectional and longitudinal analysis using two large national registries. Cancer Med. 10, 3862-3872 https://doi.org/10.1002/cam4 3938 